\title{
Thermodynamics and gauge/gravity duality for Lifshitz black holes in the presence of exponential electrodynamics
}

\author{
M. Kord Zangeneh, ${ }^{a}$ A. Dehyadegari, ${ }^{a}$ A. Sheykhi ${ }^{a, b}$ and M.H. Dehghani ${ }^{a, b}$ \\ ${ }^{a}$ Physics Department and Biruni Observatory, College of Sciences, Shiraz University, \\ Eram Square, Shiraz, P.O. Box 71454, Iran \\ ${ }^{b}$ Research Institute for Astrophysics and Astronomy of Maragha (RIAAM), \\ P.O. Box 55134-441, Maragha, Iran \\ E-mail: mkzangeneh@shirazu.ac.ir, amindehyadegari@gmail.com, \\ asheykhi@shirazu.ac.ir, mhd@shirazu.ac.ir
}

ABSTRACT: In this paper, we construct a new class of topological black hole Lifshitz solutions in the presence of nonlinear exponential electrodynamics for Einstein-dilaton gravity. We show that the reality of Lifshitz supporting Maxwell matter fields exclude the negative horizon curvature solutions except for the asymptotic AdS case. Calculating the conserved and thermodynamical quantities, we obtain a Smarr type formula for the mass and confirm that thermodynamics first law is satisfied on the black hole horizon. Afterward, we study the thermal stability of our solutions and figure out the effects of different parameters on the stability of solutions under thermal perturbations. Next, we apply the gauge/gravity duality in order to calculate the ratio of shear viscosity to entropy for a three-dimensional hydrodynamic system by using the pole method. Furthermore, we study the behavior of holographic conductivity for two-dimensional systems such as graphene. We consider linear Maxwell and nonlinear exponential electrodynamics separately and disclose the effect of nonlinearity on holographic conductivity. We indicate that holographic conductivity vanishes for $z>3$ in the case of nonlinear electrodynamics while it does not in the linear Maxwell case. Finally, we solve perturbative additional field equations numerically and plot the behaviors of real and imaginary parts of conductivity for asymptotic AdS and Lifshitz cases. We present experimental results match with our numerical ones.

KEYwords: Black Holes, Classical Theories of Gravity

ArXiv EPrint: 1601.04732 


\section{Contents}

1 Introduction 1

2 Thermodynamics of asymptotic Lifshitz solutions 4

2.1 Action and higher-dimensional solutions 4

$\begin{array}{ll}2.2 & \text { Thermodynamics of Lifshitz black holes/branes } \\ \end{array}$

$\begin{array}{lll}2.3 & \text { Thermal stability in the canonical and grand-canonical ensembles } & 12\end{array}$

3 Gauge/gravity duality 18

$\begin{array}{ll}3.1 \text { Holographic viscosity } & 18\end{array}$

$\begin{array}{lll}3.2 & \text { Holographic conductivity } & 19\end{array}$

$\begin{array}{lll}3.2 .1 & \text { Linear Maxwell case ( } \beta \text {-infinity) } & 19\end{array}$

$\begin{array}{ll}3.2 .2 & \text { Nonlinear electrodynamics } \\ 3.2 .3 & 20\end{array}$

3.2.3 Behavior of the conductivity and experimental results 21

4 Summary and concluding remarks $\quad 24$

A A brief review on gauge-gravity duality basics 25

\section{Introduction}

In the first decades of last century, physics encounters two great revolutions; one in large scale structures' domain namely special and general relativities and another in microscopic world i.e. quantum mechanics. Since quantum mechanics deals with low speed microscopic particles, physicists quickly thought about a new theory combining (at the first step) special relativity and quantum mechanics called relativistic quantum mechanics in order to describe high speed microscopic particles. In this regime, we continuously encounter production and annihilation of particles. Therefore, relativistic quantum mechanics was progressed and gave birth to a more consistent theory i.e. quantum field theory (QFT) where the Hilbert space is vast enough to include arbitrary number of particles. QFT indicates impressive theoretical results confirmed up to many significant digits by experiments in the case of weak interactions where it is allowed to perform perturbation method [1]. However, when coupling is strong, one should follow other ways in order to compute physical quantities in the context of QFT.

Fundamental particles physics is not the only place where QFT is employed. In fact, the situations where one faces non-relativistic particles produced and annihilated continuously are the other places that QFT methods can be utilized. The most familiar situations with this behavior are condensed matter systems. For instance, phonons in a lattice are produced and annihilated. Also, electrons below or above Fermi level may pass it and cause a hole 
production or annihilation. These events resemble the pair production or annihilation. Another place where QFT methods are useful is statistical field theory. When we have a system with continuous configurations, the statistical mechanics of the system is formulated in the context of statistical field theory. Accordingly, we deal with an integration in the formula of central quantity of statistical mechanics namely partition function instead of the usual summation. In the other hand, the amplitude of the transition between two states in QFT interpreted as the generating function is calculated through the path integral method and has very similar formulation to the partition function of statistical field theory. Hence, the methods of QFT can be effectively applied in these cases. A very important example of such situation happens near a critical point where we have a continuous phase transition.

As we mentioned above the common perturbation method does not work for strong interactions in QFT and hence one should look for another methods to calculate quantities in this case. One of these methods based on physics of black holes (mostly thermodynamics of black holes) is gauge/gravity duality (GGD). The first version of GGD is AdS/CFT correspondence presented by Maldacena in 1998 [2]. GGD connects the QFT (usually gauge field theories) lives on $n$-dimensional boundary of the spacetime with black hole solutions in $(n+1)$-dimensional gravity theories of the bulk. This connection is performed by equality of generating function of QFT $\left(Z_{Q F T}\right)$ and the saddle-point approximation of bulk partition function in the case of classical gravity. Through this, one can obtain the key quantity of QFT namely generating function. Computation of almost all physical quantities in QFT relies on generating function $Z_{Q F T}$.

The idea of GGD has been applied frequently in order to analyse condensed matter systems. For instance quantum Hall effect [3], fractional quantum Hall effect [4], Nernst effect [5-7] and superconductors [8-12] have been studied by this method. Furthermore, there are interesting strongly correlated electronic and atomic systems and also non-relativistic ones which possess Schrodinger symmetry [13-15]. Nevertheless, the near a critical point dynamics of such systems can be described by a relativistic conformal field theory or a more subtle scaling theory respecting the Lifshitz symmetry [16]

$$
t \rightarrow \lambda^{z} t, \quad \overrightarrow{\mathbf{x}} \rightarrow \lambda \overrightarrow{\mathbf{x}} .
$$

The spacetime which supports above symmetry on its $r$-infinity boundary is [16]

$$
d s^{2}=-\frac{r^{2 z}}{l^{2 z}} d t^{2}+\frac{l^{2} d r^{2}}{r^{2}}+r^{2} d \overrightarrow{\mathbf{x}}^{2},
$$

known as Lifshitz spacetime. In (1.2), $z$ is dynamical critical exponent. As regards the fact that thermodynamics of black holes plays a central role in GGD, the literature has encountered increasing interests on this issue. Solutions and thermodynamics of asymptotic Lifshitz black objects (usually called Lifshitz solutions) in the presence of massive gauge fields have been studied in $[17,18]$ and [19-21] respectively. Also, thermodynamics of Lifshitz solutions of gravity models containing higher curvature corrections has been considered under study [22]. Lifshitz solutions with toroidal event horizons and their thermal properties in the framework of gravity theory coupled to Abelian gauge fields with negative cosmological constant have been studied in [23]. Moreover, in Einstein-dilaton 
gravity in the presence of massless gauge fields, thermal behavior of uncharged [24] and linearly charged [25] Lifshitz black solutions have been investigated. In this context, also nonlinearly charged Lifshitz solutions with power-law Maxwell field have been taken under consideration [26]. Furthermore, thermodynamics of Gauss-Bonnet-dilaton Lifshitz black branes has been discussed in [27]. The idea of holography has been applied for systems with Lifshitz symmetry in different contexts including dilaton gravity from different points of view [28-30] and behaviors of some quantities such as viscosity [31,32] and different kinds of conductivity [12, 33-38] have been analyzed. The authors of ref. [12], constructed an Abelian Higgs model in a gravity background which is dual to a strongly coupled system at a Lifshitz-fixed point and finite temperature. They also explored the conductiviy via holographic techniques. The holographic conductivity in the presence of an additional term in gauge field action including the Weyl tensor coupled to Maxwell fied strengths in a domain-wall background whose near horizon IR geometry is Lifshitz black hole and asymptotic geometry is AdS has been calculated by performing both memberane paradigm and Kubo's formula [34]. Employing the gauge/gravity duality the holographic superconductors were explored with $z=2$ Lifshitz scaling [35]. The effects of Lifshitz dynamical exponent $z$ on the holographic superconductor models including $s$-wave and $p$-wave models have been disclosed both numerically and analytically in [36]. Via holography, superconducting phase transitions of a system dual to Yang-Mills field coupled to an axion as probes of black hole with arbitrary Lifshitz scaling have been studied with $p_{x}+i p_{y}$ condensate [37]. While the latter phase is known as unstable in the relativistic case $(z=1)$ and absence of axion field, in the non-relativistic case $(z \neq 1)$ with axion field, stability of it has been studied and behavior of Hall conductivity in non-superconducting pahase has been studied numerically as a function of Lifshitz scaling [37]. Furthermore, an analytic computation for longtitudinal DC conductivity corresponding to Lifshitz-like fixed points based on linear response theory has been performed in the presence of chiral anomalies [38] and an appropriate holographic set up has been constructed to calculate the Lifshitz sector of the DC conductivity at strong coupling and low charge density limit.

It is well-known that the behavior of conductivity have a close relation to electrodynamics model under consideration. There are several nonlinear electrodynamics models in addition to linear Maxwell one. The first one of these models is Born-Infeld electrodynamics presented in 1930's [39]. This model which arises in open superstrings and D-branes [4044], removes the divergency of charged particles' self energy. The nonlinearity of this model is determined by a parameter $\beta$ called nonlinear parameter. Born-Infeld electrodynamics recovers the linear Maxwell model for large $\beta$ 's. Recently, another nonlinear electrodynamics model has been introduced which is called exponential electrodynamics [45]. The large $\beta$ behavior of this model is similar to Born-Infeld electrodynamics. The advantage of exponential electrodynamics compared to Born-Infeld theory is that while it does not fully remove the divergency of electric field at the source, it does make this divergency much weaker in comparison with Maxwell field [46, 47]. This is more reasonable compared to the Born-Infeld case, since near the origin where $r \rightarrow 0$, the electric field of a pointlike charged particle should be an increasing function. Besides, it has been observed that the exponential nonlinear electrodynamics has crucial effects on condensation and criti- 
cal temperature of a holographic superconductor [48]. Furthermore, in recent studies on holographic superconductors, it has been shown that the exponential nonlinear electrodynamics can increase the critical values of the external magnetic field as the temperature goes to zero [49]. In this paper, we consider Einstein-dilaton gravity in the presence of exponential electrodynamics coupled to dilaton field. We construct exact asymptotically Lifshitz black hole/brane solutions and discuss the thermal behavior of them. Finally, we apply GGD to obtain viscosity and conductivity corresponding to our solutions for $(3+1)$ and $(2+1)$-dimensional systems on boundary respectively.

The layout of this paper is as follows. In section 2, we first find the exact asymptotic Lifshitz topological black hole solutions of Einstein-dilaton gravity in the presence of exponential electrodynamics coupled to dilaton field. Then, we calculate the conserved and thermodynamics quantities in order to check the satisfaction of thermodynamics first law. At the end of section 2, we disclose the effects of parameters of model on thermal stability of our solutions. In section 3, we first apply the GGD through pole method to obtain the viscosity of a system lives on three-dimensions. In continue of section 3 , we study the holographic conductivity of two-dimensional systems for both linear Maxwell and nonlinear exponential elctrodynamics. Finally, we figure out the behavior of real and imaginary parts of conductivity for asymptotic AdS and Lifshitz cases. The last section is devoted to present summary and concluding remarks.

\section{Thermodynamics of asymptotic Lifshitz solutions}

In this section, we first find asymptotic Lifsthitz solutions. Then, we study thermodynamics of our solutions and show that the first law of thermodynamics is satisfied on the horizon. Finally, we investigate thermal stability of the solutions in both canonical and grandcanonical ensembles.

\subsection{Action and higher-dimensional solutions}

The action of $(n+1)$-dimensional $(n \geq 3)$ Einstein-dilaton gravity coupled to a nonlinear electrodynamics and two linear Maxwell fields can be written as

$$
\begin{aligned}
& S=\int_{\mathcal{M}} d^{n+1} x \mathcal{L}, \\
& \mathcal{L}=\frac{\sqrt{-g}}{16 \pi}\left(\mathcal{R}-\frac{4}{n-1}(\nabla \Phi)^{2}-2 \Lambda+L(F, \Phi)-\sum_{i=1}^{2} e^{-4 /(n-1) \lambda_{i} \Phi} H_{i}\right),
\end{aligned}
$$

where $\mathcal{R}$ and $\Phi$ are Ricci scalar and dilaton scalar field, respectively. Here $F=F^{\mu \nu} F_{\mu \nu}$ and $H_{i}=\left(H_{i}\right)_{\mu \nu}\left(H_{i}\right)^{\mu \nu}$, where $F_{\mu \nu}=\partial_{[\mu} A_{\nu]},\left(H_{i}\right)_{\mu \nu}=\partial_{[\mu}\left(B_{i}\right)_{\nu]}$, and $A_{\nu}$ and $\left(B_{i}\right)_{\nu}$ are electromagnetic vector potentials. $\lambda_{i}$ and $\Lambda$ are some constants and $L(F, \Phi)$ is the Lagrangian of nonlinear electrodynamic matter source. Varying the action (2.1) with respect to the metric $g_{\mu \nu}$, scalar field $\Phi$, and electromagnetic vector potentials $A_{\nu}$ and $\left(B_{i}\right)_{\nu}$, leads 
to the following equations of motion,

$$
\begin{aligned}
& \mathcal{R}_{\mu \nu}=\frac{g_{\mu \nu}}{n-1}\left\{2 \Lambda+2 L_{F} F-L(F, \Phi)-\sum_{i=1}^{2} H_{i} e^{-4 \lambda_{i} \Phi /(n-1)}\right\} \\
&+\frac{4}{n-1} \partial_{\mu} \Phi \partial_{\nu} \Phi-2 L_{F} F_{\mu \lambda} F_{\nu}{ }^{\lambda}+2 \sum_{i=1}^{2} e^{-4 \lambda_{i} \Phi /(n-1)}\left(H_{i}\right)_{\mu \lambda}\left(H_{i}\right)_{\nu}{ }^{\lambda} \\
& \nabla^{2} \Phi+\frac{n-1}{8} L_{\Phi}+\sum_{i=1}^{2} \frac{\lambda_{i}}{2} e^{-4 \lambda_{i} \Phi /(n-1)} H_{i}=0 \\
& \nabla_{\mu}\left(L_{F} F^{\mu \nu}\right)=0 \\
& \nabla_{\mu}\left(e^{-4 \lambda_{i} \Phi /(n-1)}\left(H_{i}\right)^{\mu \nu}\right)=0
\end{aligned}
$$

where we have used the convention $X_{Y}=\partial X / \partial Y$. In this paper, we intend to consider exponential nonlinear electrodynamics [45]. The Lagrangian of such type of nonlinear electrodynamics coupled to the dilaton field in higher dimensions can be written as $[50,51]$

$$
L(F, \Phi)=4 \beta^{2} e^{4 \lambda \Phi /(n-1)}\left[\exp \left(\frac{-e^{-8 \lambda \Phi /(n-1)} F}{4 \beta^{2}}\right)-1\right]
$$

The behavior of exponential nonlinear electrodynamics coupled to dilaton field (END) for large $\beta$, namely

$$
\lim _{\beta \rightarrow \infty} L(F, \Phi)=-e^{-4 \lambda \Phi /(n-1)} F+\frac{e^{-12 \lambda \Phi /(n-1)} F^{2}}{8 \beta^{2}}+O\left(\frac{1}{\beta^{4}}\right),
$$

is similar to large $\beta$ behavior of the Born-Infeld electrodynamics coupled to dilaton field (BID)

$$
\lim _{\beta \rightarrow \infty} L_{B I}(F, \Phi)=-e^{-4 \lambda \Phi /(n-1)} F+\frac{e^{-12 \lambda \Phi /(n-1)} F^{2}}{8 \beta^{2}}+O\left(\frac{1}{\beta^{4}}\right)
$$

where

$$
L_{B I}(F, \Phi)=4 \beta^{2} e^{4 \lambda \Phi /(n-1)}\left[1-\sqrt{1+\frac{e^{-8 \lambda \Phi /(n-1)} F}{2 \beta^{2}}}\right] .
$$

This similarity makes the form chosen for END justifiable and also implies that one can consider both END and BID as the Lagrangian of nonlinear electrodynamics coupled to dilaton field.

In order to construct asymptotic Lifshitz topological black hole solutions in higher dimensions, we use the metric

$$
d s^{2}=-\frac{r^{2 z} f(r)}{l^{2 z}} d t^{2}+\frac{l^{2} d r^{2}}{r^{2} f(r)}+r^{2} d \Omega_{n-1}^{2},
$$

where $z \geq 1$ is dynamical critical exponent, $d \Omega_{n-1}^{2}$ is an $(n-1)$-dimensional hypersurface with constant curvature $(n-1)(n-2) k$ and volume $\omega_{n-1}$ and $f(r) \rightarrow 1$ as $r \rightarrow \infty$. 
Lifshitz dilaton topological black hole solutions in the presence of linear Maxwell and nonlinear power-law Maxwell fields have been studied in [25] and [52], respectively. The first term of (2.7) recovers the linear case of electrodynamic Lagrangian of [52]. Therefore, we expect that our solutions recover the results of this case. Using the metric (2.9), one can immediately integrate (2.4) and (2.5) and find

$$
\begin{aligned}
F_{r t} & =\frac{q e^{4 \lambda \Phi /(n-1)}}{r^{n-z}} \exp \left[-\frac{1}{2} L_{W}(\varrho)\right], \\
\left(H_{i}\right)_{r t} & =\frac{q_{i} e^{4 \lambda_{i} \Phi /(n-1)}}{r^{n-z}},
\end{aligned}
$$

where $\varrho \equiv q^{2} l^{2 z-2} /\left(\beta^{2} r^{2 n-2}\right)$ and $L_{W}(x)=$ Lambert $W(x)$ is the Lambert function which satisfies the identity $[53,54]$

$$
L_{W}(x) e^{L_{W}(x)}=x
$$

and has the series expansion

$$
L_{W}(x)=x-x^{2}+\frac{3}{2} x^{3}-\frac{8}{3} x^{4}+\cdots,
$$

which converges provided $|x|<1$. For $\beta \rightarrow \infty, F_{r t}$ given in eq. (2.10) reduces to the linear Maxwell case [52]

$$
\lim _{\beta \rightarrow \infty} F_{r t}=\frac{q e^{4 \lambda \Phi /(n-1)}}{r^{n-z}}-\frac{q^{3} l^{2 z-2} e^{4 \lambda \Phi /(n-1)}}{2 r^{3 n-z-2} \beta^{2}}+O\left(\frac{1}{\beta^{4}}\right) .
$$

Substituting eqs. (2.10) and (2.11) into eqs. (2.2) and (2.3), one can find the following field equations

$$
\begin{aligned}
& \frac{(n-1)^{2} r f^{\prime}+(n-1)^{2} n f+4 r^{2} f \Phi^{\prime 2}}{2(n-1) l^{2}}+\Lambda-\frac{(n-1)(n-2) k}{2 r^{2}}+\sum_{i=1}^{2} \frac{q_{i}^{2} e^{4 \lambda_{i} \Phi /(n-1)}}{l^{2(1-z)} r^{2(n-1)}}+4 \beta^{2} e^{4 \lambda \Phi /(n-1)} \Theta=0, \\
& \frac{(n-1)^{2} r f^{\prime}+(n-1)^{2}(n-2) f+2(n-1)^{2} f z-4 r^{2} f \Phi^{\prime 2}}{2(n-1) l^{2}}+\Lambda-\frac{(n-1)(n-2) k}{2 r^{2}} \\
&+\sum_{i=1}^{2} \frac{q_{i}^{2} e^{4 \lambda_{i} \Phi /(n-1)}}{l^{2(1-z)} r^{2(n-1)}}+4 \beta^{2} e^{4 \lambda \Phi /(n-1)} \Theta=0, \\
& \frac{r^{2} f^{\prime \prime}+(2 n+3 z-3) r f^{\prime}+\frac{4}{n-1} r^{2} f \Phi^{\prime 2}+\left(2 z^{2}+2(n-2) z+(n-1)(n-2)\right) f}{2 l^{2}}+\Lambda \\
&-\frac{(n-3)(n-2) k}{2 r^{2}}-\sum_{i=1}^{2} \frac{q_{i}^{2} e^{4 \lambda_{i} \Phi /(n-1)}}{l^{2(1-z)} r^{2(n-1)}}+4 \beta^{2} e^{4 \lambda \Phi /(n-1)} \Psi=0, \\
& \frac{r^{2} f^{\prime} \Phi^{\prime}+\Phi^{\prime} f r z+n r f \Phi^{\prime}+f r^{2} \Phi^{\prime \prime}}{l^{2}}-\sum_{i=1}^{2} \frac{q_{i}^{2} \lambda_{i} e^{4 \lambda_{i} \Phi /(n-1)}}{l^{2(1-z)} r^{2(n-1)}}-4 \beta^{2} \lambda e^{4 \lambda \Phi /(n-1)} \Theta=0,
\end{aligned}
$$

where

$$
\Theta=\frac{1}{2}+\frac{q l^{z-1}}{2 \beta r^{n-1}}\left(\sqrt{L_{W}(\varrho)}-\frac{1}{\sqrt{L_{W}(\varrho)}}\right)
$$


and

$$
\Psi=\frac{1}{2}-\frac{q l^{z-1}}{2 \beta r^{n-1} \sqrt{L_{W}(\varrho)}} .
$$

The solutions of eqs. (2.15)-(2.18) can be obtained as

$$
\begin{aligned}
\Phi(r)= & \frac{(n-1) \sqrt{z-1}}{2} \ln \left(\frac{r}{b}\right), \\
f(r)= & 1-\frac{m}{r^{n+z-1}}+\frac{(n-2)^{2} k l^{2}}{(n+z-3)^{2} r^{2}} \\
& -\frac{8 \beta^{2} l^{2} b^{2 z-2}}{(n-1)(n-z+1) r^{2 z-2}}\left\{\frac{1}{2}+\frac{q l^{z-1}(n-z+1)}{2 \beta} r^{z-n-1} \int r^{1-z}\left(\sqrt{L_{W}(\varrho)}-\frac{1}{\sqrt{L_{W}(\varrho)}}\right) d r\right\},
\end{aligned}
$$

where $m$ is a parameter which is related to the total mass of black hole. The above solutions will fully satisfy the field equations (2.15)-(2.18), provided

$$
\begin{aligned}
\lambda & =-\sqrt{z-1}, \quad \lambda_{1}=\frac{n-1}{\sqrt{z-1}}, \quad \lambda_{2}=\frac{n-2}{\sqrt{z-1}} \\
q_{1}^{2} & =\frac{(n+z-1)(z-1) b^{2(n-1)}}{2 l^{2 z}} \\
q_{2}^{2} & =\frac{k(n-1)(n-2)(z-1) b^{2(n-2)}}{2(z+n-3) l^{2(z-1)}}, \\
\Lambda & =-\frac{(n+z-1)(n+z-2)}{2 l^{2}}
\end{aligned}
$$

Since in the case of $k=-1, q_{2}$ is imaginary (except for $z=1$ ), we exclude this case and focus on the black hole $(k=1)$ and black brane $(k=0)$ solutions in the remaining part of this paper. It is notable to mention that althogh at first glance it seems that constants (2.21) diverge for $z=1$, one should note that $\lambda_{i} \Phi$ is finite for this case and $H_{i}=0$. Hence, for $z=1$, action (2.1) reduces to (A)dS action in the presence of a nonlinear electrodynamics field. One can perform the integration in eq. (2.20) by using MATHEMATICA software. One obtains

$$
\begin{aligned}
f(r)= & -\frac{m}{r^{n+z-1}}+\frac{(n-2)^{2} k l^{2}}{(n+z-3)^{2} r^{2}} \\
& -\frac{4 \beta^{2} l^{2} b^{2 z-2}}{(n-1)(n-z+1) r^{2 z-2}}+\frac{8 \beta^{2} l^{2} b^{2 z-2}(2 n-2)^{(z-5 n+3) /(2 n-2)}}{(z-2)^{(z+3 n-5) /(2 n-2)} r^{n+z-1}}\left(\frac{q^{2} l^{2 z-2}}{\beta^{2}}\right)^{(n-z+1) /(2 n-2)} \\
& \times\left\{4(n-1)^{2}\left[\Gamma\left(\frac{3 n+z-5}{2 n-2}, \frac{2-z}{2 n-2} L_{W}(\varrho)\right)-\Gamma\left(\frac{3 n+z-5}{2 n-2}\right)\right]\right. \\
& \left.-(z-2)^{2}\left[\Gamma\left(\frac{z-n-1}{2 n-2}, \frac{2-z}{2 n-2} L_{W}(\varrho)\right)-\Gamma\left(\frac{z-n-1}{2 n-2}\right)\right]\right\},
\end{aligned}
$$

where $\Gamma(x, y)$ and $\Gamma(x)$ are gamma functions and are related to each other with the relation

$$
\Gamma(x, y)=\Gamma(x)-\frac{y^{x}}{x} \mathbf{F}(x, 1+x,-y),
$$

where $\mathbf{F}(a, b, c)$ is the hypergeometric function [53,54]. At the first look, it seems that the function (2.22) diverges for $z=2$. However, the factor $(z-2)$ in denominator is removed 
when one uses eq. (2.23). Indeed, one can reexpress (2.22) by using relation (2.23) in terms of the hypergeometric functions as

$$
\begin{aligned}
f(r)= & 1-\frac{m}{r^{n+z-1}}+\frac{(n-2)^{2} k l^{2}}{(n+z-3)^{2} r^{2}} \\
& -\frac{4 \beta^{2} l^{2} b^{2 z-2}}{(n-1)(n-z+1) r^{2 z-2}}+\frac{4 \beta^{2} l^{2} b^{2 z-2}}{(n-1) r^{n+z-1}}\left(\frac{q^{2} l^{2 z-2}}{\beta^{2} L_{W}(\varrho)}\right)^{(n-z+1) /(2 n-2)} \\
& \times\left\{\frac{L_{W}^{2}(\varrho)}{3 n+z-5} \mathbf{F}\left(\frac{3 n+z-5}{2 n-2}, \frac{5 n+z-7}{2 n-2}, \frac{z-2}{2 n-2} L_{W}(\varrho)\right)\right. \\
& \left.+\frac{1}{n-z+1} \mathbf{F}\left(\frac{z-n-1}{2 n-2}, \frac{z+n-3}{2 n-2}, \frac{z-2}{2 n-2} L_{W}(\varrho)\right)\right\} .
\end{aligned}
$$

Using the fact that $f\left(r_{+}\right)=0$ where $r_{+}$is the outermost event horizon, one can obtain

$$
m\left(r_{+}\right)=r_{+}^{n+z-1}+\frac{(n-2)^{2} k l^{2}}{(n+z-3)^{2} r_{+}^{3-n-z}}-\frac{8 \beta^{2} l^{2} b^{2 z-2} \Xi}{(n-1)(n-z+1) r_{+}^{z-n-1}}
$$

where

$$
\begin{aligned}
\Xi= & \frac{1}{2}-\frac{(n-z+1)}{2 r_{+}^{n-z+1}}\left(\frac{q^{2} l^{2 z-2}}{\beta^{2} L_{W}\left(\varrho_{+}\right)}\right)^{(n-z+1) /(2 n-2)} \\
& \times\left\{\frac{L_{W}^{2}\left(\varrho_{+}\right)}{3 n+z-5} \mathbf{F}\left(\frac{3 n+z-5}{2 n-2}, \frac{5 n+z-7}{2 n-2}, \frac{z-2}{2 n-2} L_{W}\left(\varrho_{+}\right)\right)\right. \\
& \left.+\frac{1}{n-z+1} \mathbf{F}\left(\frac{z-n-1}{2 n-2}, \frac{z+n-3}{2 n-2}, \frac{z-2}{2 n-2} L_{W}\left(\varrho_{+}\right)\right)\right\},
\end{aligned}
$$

and $\varrho_{+}=q^{2} l^{2 z-2} /\left(\beta^{2} r_{+}^{2 n-2}\right)$. The large $\beta$ limit of $f(r)$ can be obtained by using the series expansion (2.13). The result is

$$
\begin{aligned}
f(r)= & 1-\frac{m}{r^{n+z-1}}+\frac{(n-2)^{2} k l^{2}}{(n+z-3)^{2} r^{2}} \\
& +\frac{2 q^{2} b^{2 z-2} l^{2 z}}{(n-1)(n+z-3) r^{2 n+2 z-4}}-\frac{q^{4} b^{2 z-2} l^{4 z-2}}{2(n-1)(3 n+z-5) \beta^{2} r^{4 n+2 z-6}}+O\left(\frac{1}{\beta^{4}}\right) .
\end{aligned}
$$

As we mentioned before, for $\beta \rightarrow \infty$ the NED Lagrangian reduces to linear Maxwell Lagrangian. Therefore, we expect that the large $\beta$ limit of $f(r)$ recover the metric function of topological Lifshitz black holes in Maxwell theory [52]. One should note that this is indeed the case and eq. (2.27) recover the linear case of $f(r)$ presented in [52] when $\beta \rightarrow \infty$. The behavior of $f(r)$ is depicted in figures $1 \mathrm{a}$ and $1 \mathrm{~b}$ for different values of $\beta$. Figures $1 \mathrm{a}$ and $1 \mathrm{~b}$ correspond to black brane $(k=0)$ and black hole $(k=1)$ solutions respectively. Inserting eqs. (2.19) and (2.21) into (2.10), one finds

$$
F_{r t}=\frac{q b^{2 z-2}}{r^{n+z-2}} \exp \left[-\frac{1}{2} L_{W}(\varrho)\right] .
$$

The fact that our solutions are static implies $A_{t}=A_{t}(r)$. Hence, we can obtain the gauge potential using relation $A_{t}(r)=\int F_{r t} d r$ as

$$
A_{t}=\mu+\frac{q b^{2 z-2}(2 n-2)^{(z-n-1) /(2 n-2)}}{(2-z)^{(-5+3 n+z) /(2 n-2)}}\left(\frac{q^{2} l^{2 z-2}}{\beta^{2}}\right)^{(3-n-z) /(2 n-2)}
$$




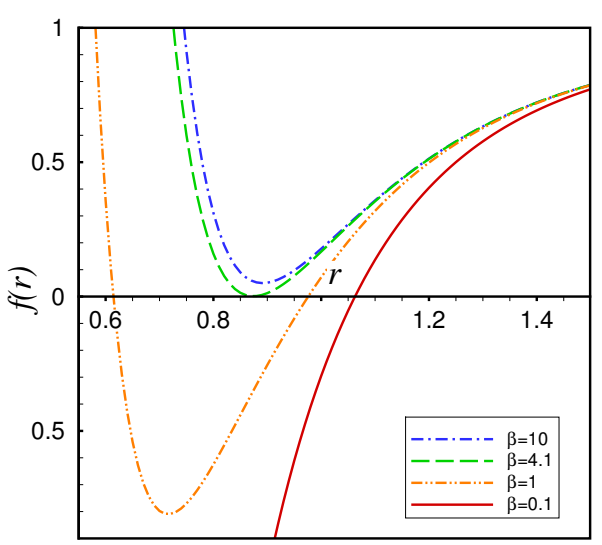

(a) $k=0, n=4$.

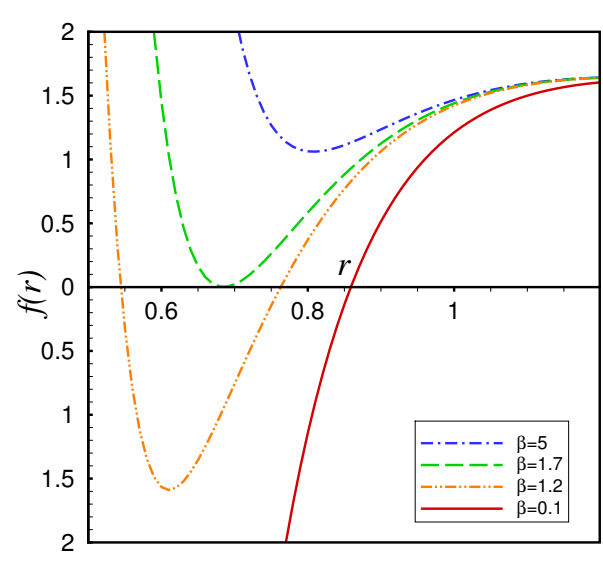

(b) $k=1, n=5$.

Figure 1. The behavior of $f(r)$ versus $r$ for $l=1.5, b=0.3, q=1.7, z=1.5$ and $m=1.6$.

$$
\begin{aligned}
& \times\left\{2(n-1)\left[\Gamma\left(\frac{3 n+z-5}{2 n-2}, \frac{2-z}{2 n-2} L_{W}(\varrho)\right)-\Gamma\left(\frac{3 n+z-5}{2 n-2}\right)\right]\right. \\
& \left.-(z-2)\left[\Gamma\left(\frac{z+n-3}{2 n-2}, \frac{2-z}{2 n-2} L_{W}(\varrho)\right)-\Gamma\left(\frac{z+n-3}{2 n-2}\right)\right]\right\}
\end{aligned}
$$

It is remarkable to note that since from one side $L_{W}(\varrho) \rightarrow 0$ as $r \rightarrow \infty$ and from another side $\Gamma(x, 0)=\Gamma(x)$, one can easily check that $A_{t}$ is finite at infinity and reduces to the constant $\mu$. Although (2.29) seems divergent for $z=2$, we can remove the factor $(z-2)$ in denominator by using (2.23) and restate $A_{t}$ as

$$
\begin{aligned}
A_{t}= & \mu-q b^{2 z-2}\left(\frac{q^{2} l^{2 z-2}}{\beta^{2} L_{W}(\varrho)}\right)^{(3-n-z) /(2 n-2)} \\
& \times\left\{\frac{L_{W}(\varrho)}{3 n+z-5} \mathbf{F}\left(\frac{3 n+z-5}{2 n-2}, \frac{5 n+z-7}{2 n-2}, \frac{z-2}{2 n-2} L_{W}(\varrho)\right)\right. \\
& \left.+\frac{1}{n+z-3} \mathbf{F}\left(\frac{n+z-3}{2 n-2}, \frac{3 n+z-5}{2 n-2}, \frac{z-2}{2 n-2} L_{W}(\varrho)\right)\right\}
\end{aligned}
$$

We require that $A_{t}$ vanishes at horizon $r=r_{+}$. Thus, $\mu$ can be calculated as

$$
\begin{aligned}
\mu= & q b^{2 z-2}\left(\frac{q^{2} l^{2 z-2}}{\beta^{2} L_{W}\left(\varrho_{+}\right)}\right)^{(3-n-z) /(2 n-2)} \\
& \times\left\{\frac{L_{W}\left(\varrho_{+}\right)}{3 n+z-5} \mathbf{F}\left(\frac{3 n+z-5}{2 n-2}, \frac{5 n+z-7}{2 n-2}, \frac{z-2}{2 n-2} L_{W}\left(\varrho_{+}\right)\right)\right. \\
& \left.+\frac{1}{n+z-3} \mathbf{F}\left(\frac{n+z-3}{2 n-2}, \frac{3 n+z-5}{2 n-2}, \frac{z-2}{2 n-2} L_{W}\left(\varrho_{+}\right)\right)\right\} .
\end{aligned}
$$

In the next subsection we study thermodynamics of the solutions by computing thermodynamic and conserved quantities. 


\subsection{Thermodynamics of Lifshitz black holes/branes}

In this section, we study thermodynamics of lifshitz black hole and brane solutions. Since, the mass is a fundamental quantity in studying the thermodynamics, we start with its calculation. Using the modified subtraction method of Brown and York (BY) [55], one can find mass per unit volume $\omega_{n-1}$ as (see ref. [52])

$$
M=\frac{(n-1) m}{16 \pi l^{z+1}},
$$

where $m$ is the geometrical mass given by (2.25) in terms of outer horizon radius $r_{+}$. Using the Gauss law, one can also compute the charge as

$$
Q=\frac{1}{4 \pi} \int r^{n-1} L_{F} F_{\mu \nu} n^{\mu} u^{\nu} d \Omega,
$$

where $n^{\mu}$ and $u^{\nu}$ are the unit spacelike and timelike normals to a sphere of radius $r$ given as

$$
n^{\mu}=\frac{1}{\sqrt{-g_{t t}}} d t=\frac{l^{z}}{r^{z} \sqrt{f(r)}} d t, \quad u^{\nu}=\frac{1}{\sqrt{g_{r r}}} d r=\frac{r \sqrt{f(r)}}{l} d r .
$$

Therefore, the charge per unit volume $\omega_{n-1}$ is

$$
Q=\frac{q l^{z-1}}{4 \pi} .
$$

The electric potential $U$ is defined as

$$
U=\left.A_{\mu} \chi^{\mu}\right|_{r \rightarrow \infty}-\left.A_{\mu} \chi^{\mu}\right|_{r=r_{+}},
$$

where $\chi=\partial_{t}$ is the null generator of the horizon. Therefore, it is a matter of calculation to obtain $U$ by using (2.30) as

$$
\begin{aligned}
U= & q b^{2 z-2}\left(\frac{q^{2} l^{2 z-2}}{\beta^{2} L_{W}\left(\varrho_{+}\right)}\right)^{(3-n-z) /(2 n-2)} \\
& \times\left\{\frac{L_{W}\left(\varrho_{+}\right)}{3 n+z-5} \mathbf{F}\left(\frac{3 n+z-5}{2 n-2}, \frac{5 n+z-7}{2 n-2}, \frac{z-2}{2 n-2} L_{W}\left(\varrho_{+}\right)\right)\right. \\
& \left.+\frac{1}{n+z-3} \mathbf{F}\left(\frac{n+z-3}{2 n-2}, \frac{3 n+z-5}{2 n-2}, \frac{z-2}{2 n-2} L_{W}\left(\varrho_{+}\right)\right)\right\} .
\end{aligned}
$$

The area law of the black hole entropy states that the entropy of a black hole is the quarter of event horizon area [56-58]. The entropy of almost all kinds of black holes in Einstein gravity including dilaton black holes is calculated by using this near universal law [59-62]. Thus, the entropy per unit volume $\omega_{n-1}$ of the Lifshitz black holes can be found as

$$
s=\frac{r_{+}^{n-1}}{4} .
$$

The Hawking temperature is

$$
T=\frac{r_{+}^{z+1} f^{\prime}\left(r_{+}\right)}{4 \pi l^{z+1}},
$$


which can be calculated as

$$
\begin{aligned}
T= & \frac{(n+z-1) r_{+}^{z}}{4 \pi l^{z+1}}+\frac{(n-2)^{2} k l^{1-z}}{4 \pi(n+z-3) r_{+}^{2-z}} \\
& -\frac{2 \beta^{2} l^{1-z} b^{2 z-2}}{\pi(n-1) r_{+}^{z-2}}\left[\frac{1}{2}+\frac{q l^{z-1}}{2 \beta r_{+}^{n-1}}\left(\sqrt{L_{W}\left(\varrho_{+}\right)}-\frac{1}{\sqrt{L_{W}\left(\varrho_{+}\right)}}\right)\right] .
\end{aligned}
$$

The large $\beta$ limit of temperature reproduces the temperature of Einstein-Maxwell dilaton Lifshitz black holes [52]:

$$
T=\frac{(n+z-1) r_{+}^{z}}{4 \pi l^{z+1}}+\frac{(n-2)^{2} k l^{1-z}}{4 \pi(n+z-3) r_{+}^{2-z}}-\frac{q^{2} l^{z-1} b^{2 z-2}}{2 \pi(n-1) r_{+}^{2 n+z-4}}+\frac{q^{4} l^{3 z-3} b^{2 z-2}}{8 \pi(n-1) r_{+}^{4 n+z-6} \beta^{2}}+O\left(\frac{1}{\beta^{4}}\right)
$$

as one expects. We need the Smarr-type mass formula in order to check the satisfaction of first law of thermodynamics. Using (2.25), (2.32), (2.34) and (2.37), the Smarr-type mass can be written as

$$
\begin{aligned}
M(s, Q)= & \frac{(n-1)(4 s)^{(n+z-1) /(n-1)}}{16 \pi l^{z+1}}+\frac{(n-1)(n-2)^{2} k(4 s)^{(n+z-3) /(n-1)}}{16 \pi l^{z-1}(n+z-3)^{2}} \\
& -\frac{\beta^{2} b^{2 z-2}(4 s)^{(n-z+1) /(n-1)} \Pi}{2 \pi l^{z-1}(n-z+1)}
\end{aligned}
$$

where

$$
\begin{aligned}
\Pi= & \frac{1}{2}-\frac{(n-z+1)}{2(4 s)^{(n-z+1) /(n-1)}}\left(\frac{16 \pi^{2} Q^{2}}{\beta^{2} L_{W}(\zeta)}\right)^{(n-z+1) /(2 n-2)} \\
& \times\left\{\frac{L_{W}(\zeta)^{2}}{3 n+z-5} \mathbf{F}\left(\frac{3 n+z-5}{2 n-2}, \frac{5 n+z-7}{2 n-2}, \frac{z-2}{2 n-2} L_{W}(\zeta)\right)\right. \\
& \left.+\frac{1}{n-z+1} \mathbf{F}\left(\frac{z-n-1}{2 n-2}, \frac{n+z-3}{2 n-2}, \frac{z-2}{2 n-2} L_{W}(\zeta)\right)\right\}
\end{aligned}
$$

and $\zeta=\pi^{2} Q^{2} /\left(\beta^{2} s^{2}\right)$. As $\beta \rightarrow \infty$, the behavior of Smarr-type mass is

$$
\begin{aligned}
M(s, Q)= & \frac{(n-1)(4 s)^{(n+z-1) /(n-1)}}{16 \pi l^{z+1}}+\frac{(n-1)(n-2)^{2} k(4 s)^{(n+z-3) /(n-1)}}{16 \pi(n+z-3)^{2} l^{z-1}} \\
& +\frac{2 \pi Q^{2} b^{2 z-2}(4 s)^{(3-n-z) /(n-1)}}{(n+z-3) l^{z-1}}-\frac{8 \pi^{3} Q^{4} b^{2 z-2}(4 s)^{(5-3 n-z) /(n-1)}}{(3 n+z-5) l^{z-1} \beta^{2}}+O\left(\frac{1}{\beta^{2}}\right) .
\end{aligned}
$$

This satisfy our expectation that the mass of the linear Maxwell case should be recovered [52]. Now, in order to check the satisfaction of the first law of thermodynamics, we take $S$ and $Q$ as a complete set of extensive quantities for mass $M(s, Q)$. Then, we define their conjugate intensive quantities as temperature $T$ and electric potential $U$ that implies

$$
T=\left(\frac{\partial M}{\partial s}\right)_{Q} \quad \text { and } \quad U=\left(\frac{\partial M}{\partial Q}\right)_{s} .
$$

Intensive quantities computed by (2.44) are in coincidance with ones obtained by (2.36) and (2.39). Hence, the first law of thermodynamics

$$
d M=T d s+U d Q .
$$




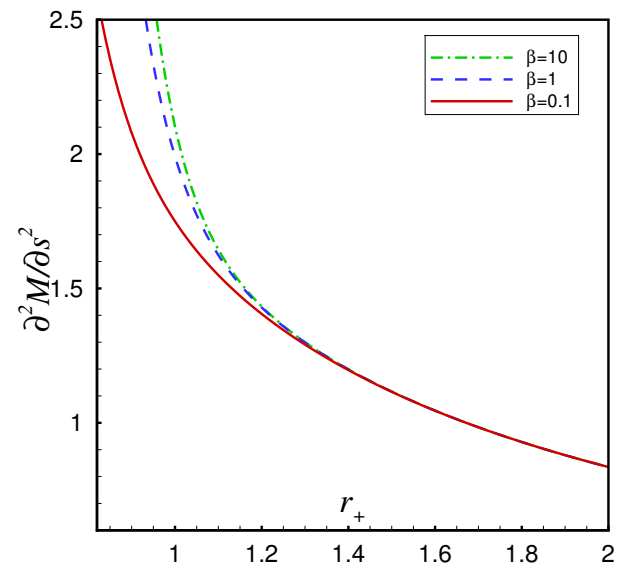

(a)

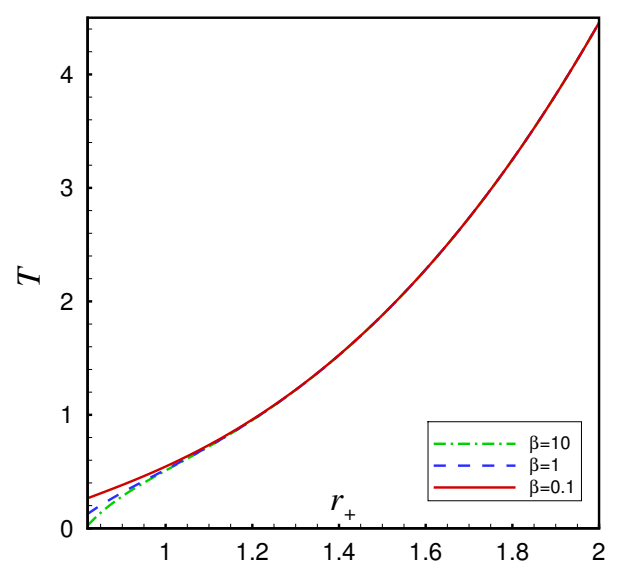

(b)

Figure 2. The behaviors of $\left(\partial^{2} M / \partial s^{2}\right)_{Q}$ and $T$ versus $r_{+}$for $k=0$ with $l=b=1, q=1.1, z=3$ and $n=5$.

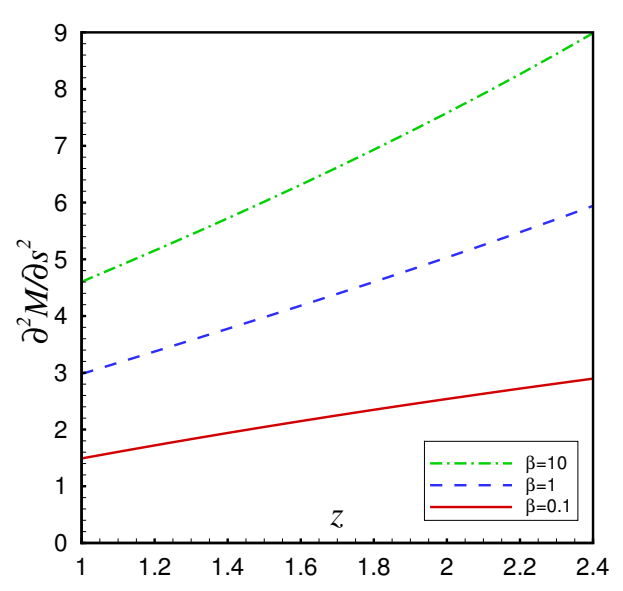

(a)

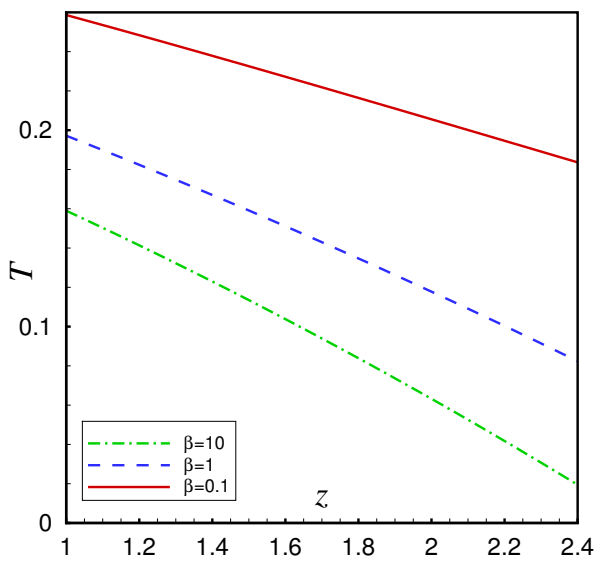

(b)

Figure 3. The behaviors of $\left(\partial^{2} M / \partial s^{2}\right)_{Q}$ and $T$ versus $z$ for $k=0$ with $l=b=1, q=0.5$, $r_{+}=0.7$ and $n=5$.

is satisfied. In the next subsection we investigate the stability of this thermodynamic system under thermal perturbations.

\subsection{Thermal stability in the canonical and grand-canonical ensembles}

In the first part of this section, we showed that one can regard a black hole solution as a thermodynamic system. On the other hand, it is necessary to investigate the stability of a thermodynamic system under thermal perturbations. Therefore, this subsection is devoted to study the thermal stability of the obtained solutions of the previous sections. The formal way to analyze the stability of a thermodynamic system with respect to small 


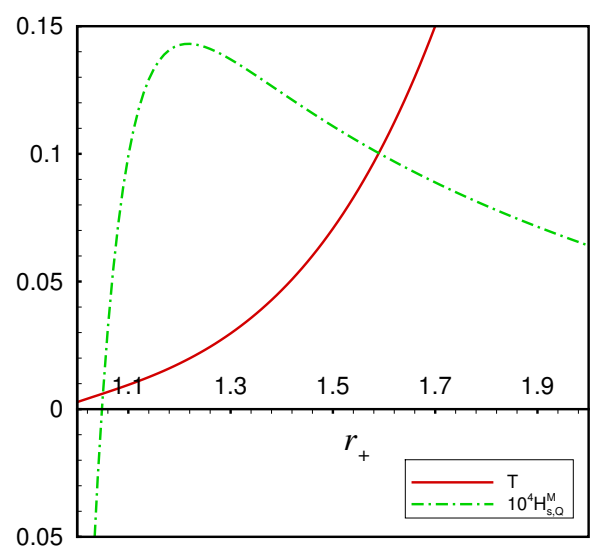

Figure 4. The behavior of $10^{4} \mathbf{H}_{s, Q}^{M}$ and $T$ versus $r_{+}$for $k=0$ with $l=2, b=1, q=0.2, n=5$, $z=6$ and $\beta=0.1$.

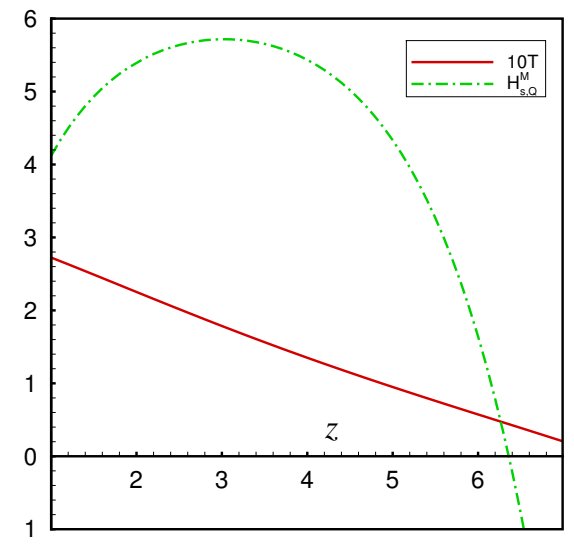

Figure 5. The behavior of $\mathbf{H}_{s, Q}^{M}$ and $10 T$ versus $z$ for $k=0$ with $l=b=1, q=0.2, n=5$, $r_{+}=0.7$ and $\beta=0.1$.

variations of the thermodynamic coordinates is by investigating the behavior of the entropy $s(M, Q)$ around equilibrium. This analysis can also be performed in terms of the Legendre transformation of entropy namely $M(s, Q)$. In any ensemble, the local stability requires that the energy $M(s, Q)$ be a convex function of its extensive variable [63-65]. The number of thermodynamic variables is ensemble-dependent. In the canonical ensemble, since the charge is fixed, the positivity of $\left(\partial^{2} M / \partial s^{2}\right)_{Q}$ in the ranges where temperature $T$ is positive suffices to ensure the local stability. For the Lifshitz black solutions we have,

$$
\begin{aligned}
\left(\frac{\partial^{2} M}{\partial s^{2}}\right)_{Q}= & \frac{z(n-1+z) r_{+}^{z-n+1}}{(n-1) \pi l^{z+1}}+\frac{k(n-2)^{2}(z-2) r_{+}^{z-n-1}}{\pi l^{z-1}(n-1)(z+n-3)} \\
& +\frac{8(z-2) \beta^{2} r_{+}^{3-n-z}}{\pi(n-1)^{2} l^{z-1} b^{2-2 z}}\left\{\frac{1}{2}+\frac{q l^{z-1} r_{+}^{1-n}}{2 \beta(z-2)}\left[(n+z-3) \sqrt{L_{W}\left(\varrho_{+}\right)}-\frac{z-2}{\sqrt{L_{W}\left(\varrho_{+}\right)}}\right]\right\} .
\end{aligned}
$$




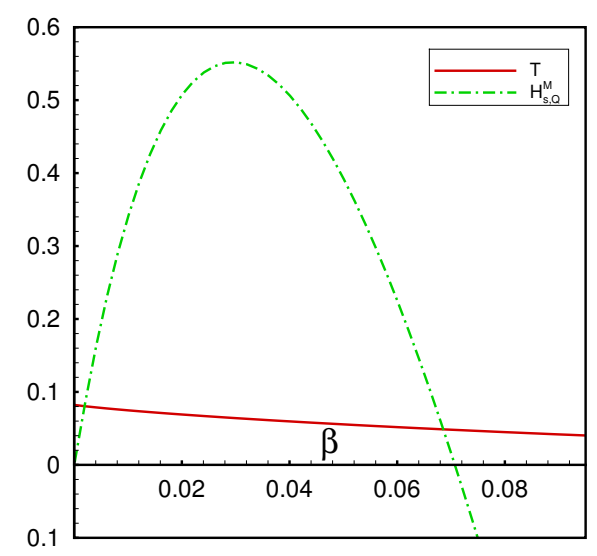

Figure 6. The behavior of $\mathbf{H}_{s, Q}^{M}$ and $T$ versus $\beta$ for $k=0$ with $l=b=1, q=0.2, n=5, z=6.5$ and $r_{+}=0.7$.

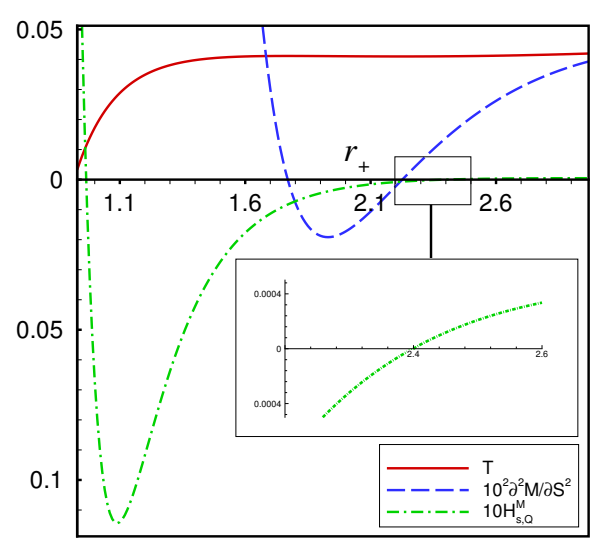

Figure 7. The behavior of $10^{2}\left(\partial^{2} M / \partial s^{2}\right)_{Q}, 10 \mathbf{H}_{s, Q}^{M}$ and $T$ versus $r_{+}$for $k=1$ with $l=7, b=1$, $q=0.5, n=4, z=1.5$ and $\beta=3$.

The behavior of (2.46) for large $\beta$ is

$$
\begin{aligned}
\left(\frac{\partial^{2} M}{\partial s^{2}}\right)_{Q}= & \frac{z(n-1+z) r_{+}^{z-n+1}}{(n-1) \pi l^{z+1}}+\frac{k(n-2)^{2}(z-2) r_{+}^{z-n-1}}{\pi l^{z-1}(n-1)(z+n-3)}+\frac{2 q^{2}(2 n+z-4) b^{2 z-2} r_{+}^{5-3 n-z}}{\pi(n-1)^{2} l^{1-z}} \\
& -\frac{q^{4}(4 n+z-6) b^{2 z-2} r_{+}^{7-5 n-z}}{2 \pi(n-1)^{2} l^{3-3 z} \beta^{2}}+O\left(\frac{1}{\beta^{4}}\right)
\end{aligned}
$$

which recovers the result of [52] for the linear Maxwell electrodynamics, as expected. Before we turn to grand canonical case it is remarkable to find $\left(\partial^{2} M / \partial s^{2}\right)_{Q}$ for highly nonlinear case i.e. $\beta \rightarrow 0$. In this case the behavior of $\left(\partial^{2} M / \partial s^{2}\right)_{Q}$ is

$$
\lim _{\beta \rightarrow 0}\left(\frac{\partial^{2} M}{\partial s^{2}}\right)_{Q}=\frac{z(n-1+z) r_{+}^{z-n+1}}{(n-1) \pi l^{z+1}}+\frac{k(n-2)^{2}(z-2) r_{+}^{z-n-1}}{\pi l^{z-1}(n-1)(z+n-3)}
$$




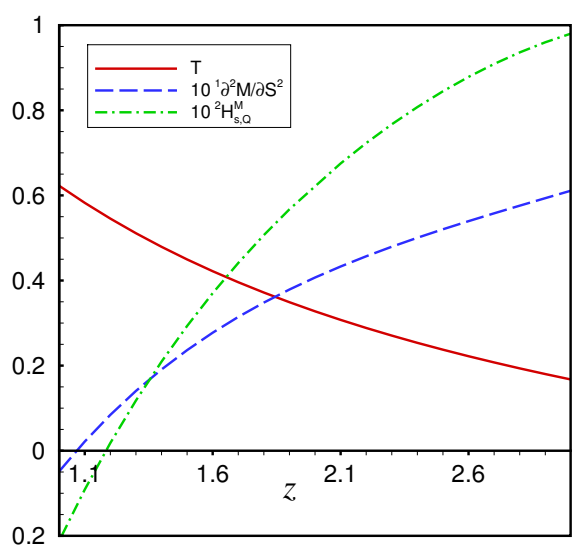

Figure 8. The behavior of $10^{-1}\left(\partial^{2} M / \partial s^{2}\right)_{Q}, 10^{-2} \mathbf{H}_{s, Q}^{M}$ and $T$ versus $z$ for $k=1$ with $l=b=1$, $q=0.1, n=5, r_{+}=0.6$ and $\beta=3$.

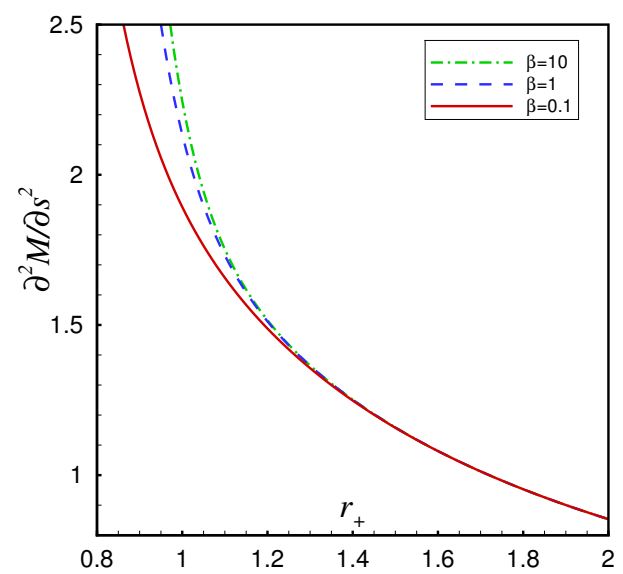

(a)

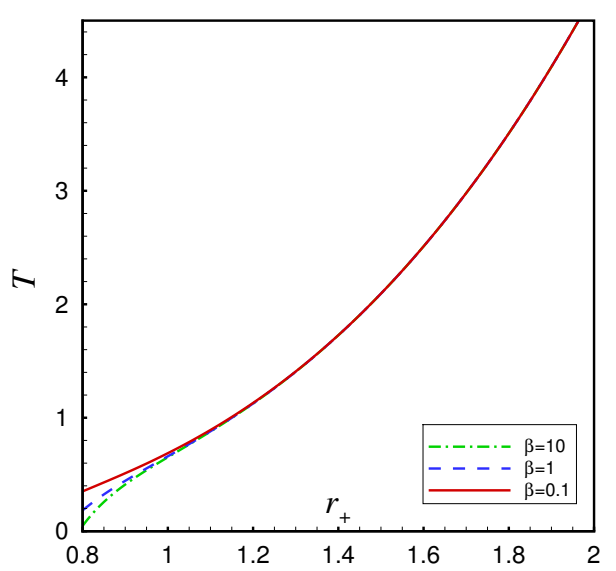

(b)

Figure 9. The behaviors of $\left(\partial^{2} M / \partial s^{2}\right)_{Q}$ and $T$ versus $r_{+}$for $k=1$ with $l=b=1, q=1.1, z=3$ and $n=5$.

$$
+\frac{4 q \beta r_{+}^{4-2 n-z}(n+z-3)}{\pi(n-1)^{2} b^{2-2 z}} \sqrt{L_{W}\left(\frac{q^{2} l^{2 z-2}}{\beta^{2} r_{+}^{2 n-2}}\right)}
$$

Soon we use (2.48) to discuss thermal stability of highly nonlinear solutions. In the grandcanonical ensemble, since $Q$ is not a fixed parameter, the local stability requires the positivity of determinant of Hessian matrix $\mathbf{H}_{s Q}^{M}=\left[\partial^{2} M / \partial s \partial Q\right]>0$. The determinant of the Hessian matrix $\mathbf{H}_{s, Q}^{M}$ for the solutions under consideration can be calculated as

$$
\mathbf{H}_{s, Q}^{M}=\frac{4 \beta b^{2 z-2} l^{3-3 z}}{q(n-1)^{2} r_{+}^{n-3}} \sqrt{L_{W}\left(\eta_{+}\right)}\left[\Upsilon+\frac{4 q \beta(z-2) b^{2 z-2} l^{z-1}}{(n-1) r_{+}^{n+2 z-3}}\left(\sqrt{L_{W}\left(\eta_{+}\right)}-\frac{1}{\sqrt{L_{W}\left(\eta_{+}\right)}}\right)\right]
$$




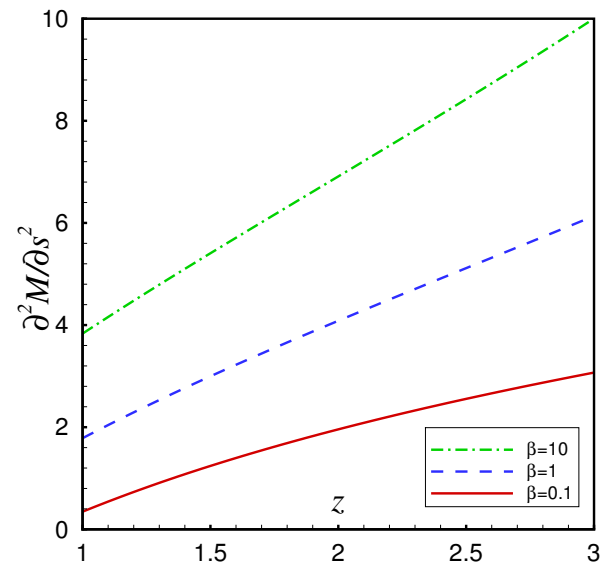

(a)

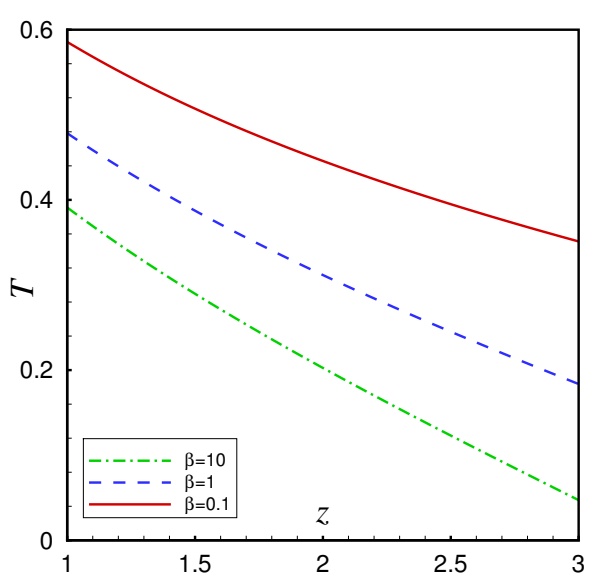

(b)

Figure 10. The behaviors of $\left(\partial^{2} M / \partial s^{2}\right)_{Q}$ and $T$ versus $z$ for $k=1$ with $l=b=1, q=1.1$, $r_{+}=0.8$ and $n=5$.

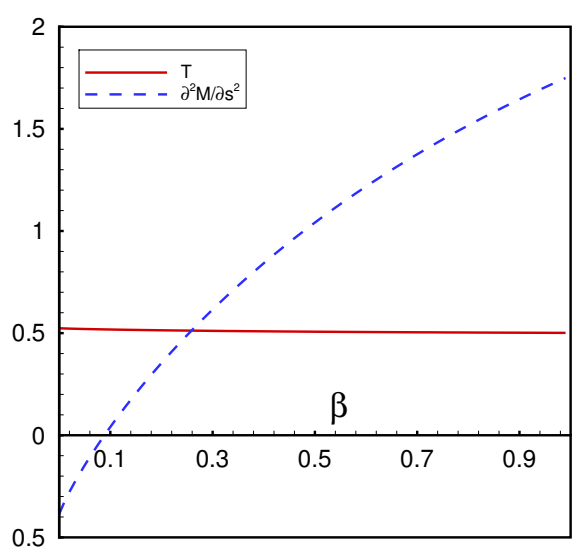

Figure 11. The behavior of $\left(\partial^{2} M / \partial s^{2}\right)_{Q}$ and $T$ versus $\beta$ for $k=1$ with $l=1, b=0.3, q=0.1$, $n=5, r_{+}=0.5$ and $z=1.3$.

$$
\begin{aligned}
& -\frac{16 b^{2 z-2} l^{2-2 z}(z-2)}{r_{+}^{n-z-1}(2 n-2)^{2}}\left(\frac{\beta^{2} L_{W}\left(\eta_{+}\right)}{q^{2} l^{2 z-2}}\right)^{(n+z-3) /(2 n-2)} \\
& \times\left[\Upsilon+\frac{4 q \beta b^{2 z-2} l^{z-1}}{(n-1) r_{+}^{n+2 z-3}}\left((n+z-3) \sqrt{L_{W}\left(\eta_{+}\right)}-\frac{z-2}{\sqrt{L_{W}\left(\eta_{+}\right)}}\right)\right] \\
& \times\left\{\frac{L_{W}\left(\eta_{+}\right)}{3 n+z-5} \mathbf{F}\left(\frac{3 n+z-5}{2 n-2}, \frac{5 n+z-7}{2 n-2}, \frac{z-2}{2 n-2} L_{W}(\zeta)\right)\right. \\
& \left.+\frac{1}{n+z-3} \mathbf{F}\left(\frac{n+z-3}{2 n-2}, \frac{3 n+z-5}{2 n-2}, \frac{z-2}{2 n-2} L_{W}(\zeta)\right)\right\},
\end{aligned}
$$




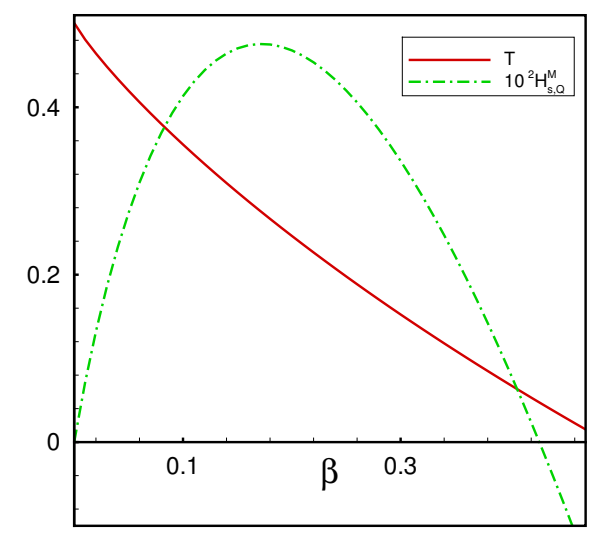

Figure 12. The behavior of $10^{-2} \mathbf{H}_{s, Q}^{M}$ and $T$ versus $\beta$ for $k=1$ with $l=0.72, b=0.8, q=0.4$, $n=6, r_{+}=0.5$ and $z=3$.

where

$$
\Upsilon=\frac{z(n+z-1)}{l^{2}}+\frac{(z-2)(n-2)^{2} k}{(n+z-3) r_{+}^{2}}+\frac{4(z-2) \beta^{2} b^{2 z-2}}{(n-1) r_{+}^{2 z-2}} .
$$

The large $\beta$ behavior of $\mathbf{H}_{s Q}^{M}$ is

$$
\begin{aligned}
\mathbf{H}_{s, Q}^{M}= & \frac{4 z(n+z-1) b^{2 z-2} r_{+}^{4-2 n}}{(n-1)(n+z-3) l^{2 z}}+\frac{4(n-2)^{2}(z-2) k b^{2 z-2} r_{+}^{2-2 n}}{(n-1)(n+z-3)^{2} l^{2 z-2}} \\
& -\frac{8 q^{2}(z-2) b^{4 z-4} r_{+}^{8-4 n-2 z}}{(n-1)^{2}(n+z-3)}+\frac{2 q^{2} b^{2 z-2}}{(n-1)(3 n+z-5) \beta^{2} r_{+}^{4 n-4}} \\
& \times\left(\frac{q^{2}(z-2) b^{2 z-2} l^{2 z-2}}{(n-1)(n+z-3) r_{+}^{2 n+2 z-6}}-\frac{3 k(n-2)^{3}}{n+z-3}-\frac{3 z(n+z-1) r_{+}^{2}}{l^{2}}\right)+O\left(\frac{1}{\beta^{4}}\right),
\end{aligned}
$$

which coincides with the one of linear Maxwell case [52]. In what follows we discuss the stability for $k=0$ (black branes) and $k=1$ (spherical black holes), separately.

$\boldsymbol{k}=\mathbf{0}: \quad$ in this case, calculations show that the solutions are always stable in the canonical ensemble. Although it is difficult to see this for arbitrary $\beta$ from (2.46), we can show this fact for large and small $\beta$ 's. For large $\beta$ where the linear Maxwell regime is dominant, this fact that the solutions are always stable in canonical ensemble has been pointed out in [52]. For small $\beta$, the behaviour of $\left(\partial^{2} M / \partial s^{2}\right)_{Q}$ has been given in (2.48). It is obvious from (2.48) that $\lim _{\beta \rightarrow 0}\left(\partial^{2} M / \partial s^{2}\right)_{Q}>0$ for $k=0$. Figures 2a and 3a depict the fact that black branes are always stable in canonical ensemble. Of course, one should check the positivity of temperature for these choices. This can be seen in figures $2 \mathrm{~b}$ and $3 \mathrm{~b}$ which show that the temperature is positive for these choices and therefore we have black branes. In grand canonical ensemble, the system is thermally stable provided that the radius of the black hole is larger than $r_{+\min }$ (figure 4 ). Figure 5 shows that there is a $z_{\max }$ that for values greater than it we encounter instability. We also have a $\beta_{\max }$ that black branes are stable under thermal perturbations for values lower than it as one can see in figure 6 . 
$\boldsymbol{k}=\mathbf{1}:$ as one can see from figure 7, there is a Hawking-Page phase transition between small and large black holes in both ensembles. In terms of $z$ we have an ensemble-dependent minimum $z_{\text {min }}$ which depends on the parameters of the system and black holes are unstable for values lower than it as figure 8 shows. The value of $z_{\min }$ is greater in grand-canonical ensemble compare to that in canonical ensemble. For highly nonlinear case (small $\beta$ ) and also linear case (large $\beta$ ) black holes are stable for $z \geq 2$ in canonical ensemble as one can see from (2.47) and (2.48). Hence, one can conclude that there are always stable black holes for $z \geq 2$ in canonical ensemble (figures 9 and 10). Figure 11 shows that black holes are unstable in canonical ensemble for $\beta<\beta_{\min }$ in the case of $z<2$. In grand-canonical ensemble we have stable black holes for $\beta<\beta_{\max }$ (figure 12).

\section{Gauge/gravity duality}

In this section, we would like to perform the gauge/gravity duality idea on our obtained solutions. We first try to calculate zero-frequency shear viscosity for a hydrodynamic system. Then, we employ this idea to study the behavior of the holographic conductivity for a two dimensional system for both asymptotic AdS $(z=1)$ and asymptotic Lifshitz cases and present experimental observations matched with obtained results.

\subsection{Holographic viscosity}

Here we intend to calculate the ratio of shear viscosity to entropy $\eta / s$ in the zero frequency limit. For this aim, we use the pole method [66, 67]. We consider the five-dimensional planar metric

$$
d s^{2}=-\frac{r^{2 z} f(r)}{l^{2 z}} d t^{2}+\frac{l^{2} d r^{2}}{r^{2} f(r)}+r^{2}\left(d x_{1}^{2}+d x_{2}^{2}+d x_{3}^{2}\right),
$$

where the QFT lives on the 4-dimensional $r$-infinity boundary. Defining $y=1-r_{+}^{2} / r^{2}$, one can rewrite (3.1) as

$$
d s^{2}=-\frac{F(y) r_{+}^{2 z}}{l^{2 z}(1-y)^{z}} d t^{2}+\frac{l^{2} d y^{2}}{4 F(y)(1-y)^{2}}+\frac{r_{+}^{2}}{1-y}\left(d x_{1}^{2}+d x_{2}^{2}+d x_{3}^{2}\right),
$$

where

$$
\begin{aligned}
F(y)= & 1-\frac{m(1-y)^{(3+z) / 2}}{r_{+}^{3+z}}-\frac{4 \beta^{2} l^{2} b^{2 z-2}(1-y)^{z-1}}{3(5-z) r_{+}^{2 z-2}}+\frac{4 \beta^{2} l^{2} b^{2 z-2}(1-y)^{(3+z) / 2}}{3 r_{+}^{3+z}}\left(\frac{q^{2} l^{2 z-2}}{\beta^{2} L_{W}\left(\varrho_{y}\right)}\right)^{(5-z) / 6} \\
& \times\left\{\frac{L_{W}^{2}\left(\varrho_{y}\right)}{7+z} \mathbf{F}\left(\frac{7+z}{6}, \frac{13+z}{6}, \frac{z-2}{6} L_{W}\left(\varrho_{y}\right)\right)+\frac{1}{5-z} \mathbf{F}\left(\frac{z-5}{6}, \frac{z+1}{6}, \frac{z-2}{6} L_{W}\left(\varrho_{y}\right)\right)\right\}
\end{aligned}
$$

$\varrho_{y} \equiv q^{2} l^{2 z-2}(1-y)^{3} /\left(\beta^{2} r_{+}^{6}\right)$ and horizon located at $y=0$. In order to use the pole method, we first apply an off-shell perturbation

$$
d x_{i} \rightarrow d x_{i}+\varepsilon e^{-i \omega t} d x_{j}
$$

where $\varepsilon$ is an infinitesimal positive parameter. Then, by using the perturbed metric and the expansion of $F(y)$ near the horizon, namely

$$
F(y)=\left[\frac{z+3}{2}-\frac{2 \beta^{2} b^{2 z-2} l^{2}}{3 r_{+}^{2 z-2}}-\frac{2 q \beta b^{2 z-2} l^{z+1}}{3 r_{+}^{2 z+1}}\left(\sqrt{L_{W}\left(\varrho_{+}\right)}-\frac{1}{\sqrt{L_{W}\left(\varrho_{+}\right)}}\right)\right] y
$$




$$
\begin{aligned}
& -\left[\frac{(z+3)(z+1)}{8}-\frac{(3 z+2) q^{2} b^{2 z-2} l^{2 z}}{6 r_{+}^{2 z+4}} \exp \left[-\frac{1}{2} L_{W}\left(\varrho_{+}\right)\right]\right. \\
& \left.+\frac{(3 z-1) \beta^{2} b^{2 z-2} l^{2}}{6 r_{+}^{2 z-2}}\left(\exp \left[-\frac{1}{2} L_{W}\left(\varrho_{+}\right)\right]-1\right)\right] y^{2}+O\left(y^{3}\right),
\end{aligned}
$$

we calculate the residue of the pole at $y=0$ in the Lagrangian density

$$
\operatorname{Res}_{y=0} \mathcal{L}=-\frac{\varepsilon^{2} \omega^{2} r_{+}^{3} e^{-2 i \omega t}}{128 \pi^{2} T}
$$

where $T$ is given by eq. (2.39). Now, via the formula $[66,67]$

$$
\eta=-8 \pi T \lim _{\varepsilon, \omega \rightarrow 0} \frac{\operatorname{Res}_{y=0} \mathcal{L}}{\varepsilon^{2} \omega^{2}},
$$

the shear viscosity for zero frequency case can be obtained as

$$
\eta=\frac{r_{+}^{3}}{16 \pi}
$$

Therefore, the ratio $\eta / s$ is obtained as

$$
\frac{\eta}{s}=\frac{1}{4 \pi}
$$

where we have used eq. (2.37). Therefore, we regain the well-known value $1 / 4 \pi$ for $\eta / s$ as many cases in Einstein gravity. This implies that neither non-AdS symmetry of the system on boundary nor the presence of the dilaton and additional gauge fields cannot affect the value of the shear viscosity of the system.

\subsection{Holographic conductivity}

Our aim in this subsection is to calculate the holographic conductivity by performing gauge/gravity duality for both linear Maxwell (infinite $\beta$ ) and nonlinear cases. Finally, we depict the behaviour of conductivity for linear and nonlinear electrodynamics cases and show that our results are supported by some experimental observations.

\subsubsection{Linear Maxwell case ( $\beta$-infinity)}

We consider a four-dimensional planar metric

$$
d s^{2}=-\frac{\mathcal{F}_{\infty}(u) r_{+}^{4 z}}{l^{2 z} u^{2 z}} d t^{2}+\frac{l^{2} d u^{2}}{\mathcal{F}_{\infty}(u) u^{2}}+\frac{r_{+}^{4}}{u^{2}}\left(d x_{1}^{2}+d x_{2}^{2}\right),
$$

where

$$
\mathcal{F}_{\infty}(u)=1-\frac{m u^{z+2}}{r_{+}^{2 z-4}}+\frac{q^{2} b^{2 z-2} l^{2 z} u^{2 z+2}}{z r_{+}^{4 z+4}}
$$

which can be obtained by defining $u=r_{+}^{2} / r$ in the metric (2.9). The horizon locates at $u=r_{+}$, while the QFT lives at $u=0$ boundary. We turn on perturbations $g_{t x_{1}}(u) e^{-i \omega t}$ 
and $A_{x_{1}}(u) e^{-i \omega t}$ and therefore receive one Einstein and one electrodynamic additional equations of motion as

$$
\begin{gathered}
A_{x_{1}}^{\prime \prime}(u)+\left[\frac{\mathcal{F}_{\infty}^{\prime}(u)}{\mathcal{F}_{\infty}(u)}+\frac{3(1-z)}{u}\right] A_{x_{1}}^{\prime}(u)+ \\
\frac{l^{2 z+2} u^{2 z-2}}{r_{+}^{4 z} \mathcal{F}_{\infty}(u)^{2}}\left[\omega^{2} A_{x_{1}}(u)-\frac{q b^{2 z-2} u^{z} \mathcal{F}_{\infty}(u)}{l^{2} r_{+}^{2 z}}\left(2 g_{t x_{1}}(u)+u g_{t x_{1}}^{\prime}(u)\right)\right]=0
\end{gathered}
$$

and

$$
2 g_{t x_{1}}(u)+u g_{t x_{1}}^{\prime}(u)=4 q r_{+}^{2 z-4} u^{2-z} A_{x_{1}}(u),
$$

where the prime denotes derivative with respect to $u$. Combining eqs. (3.10) and (3.11), one can easily find a decoupled equation for $A_{x_{1}}$

$$
A_{x_{1}}^{\prime \prime}(u)+\left[\frac{\mathcal{F}_{\infty}^{\prime}(u)}{\mathcal{F}_{\infty}(u)}+\frac{3(1-z)}{u}\right] A_{x_{1}}^{\prime}(u)+\frac{l^{2 z+2} u^{2 z-2}}{r_{+}^{4 z} \mathcal{F}_{\infty}(u)^{2}}\left[\omega^{2}-\frac{4 q^{2} b^{2 z-2} u^{2} \mathcal{F}_{\infty}(u)}{l^{2} r_{+}^{4}}\right] A_{x_{1}}(u)=0 .
$$

Near the boundary $u=0$, eq. (3.12) reduces approximately to

$$
A_{x_{1}}^{\prime \prime}(u)+\frac{3(1-z)}{u} A_{x_{1}}^{\prime}(u)+\cdots=0,
$$

which has the solution

$$
A_{x_{1}}(u)=A^{0}+A^{1} u^{3 z-2}+\cdots,
$$

where $A^{0}$ and $A^{1}$ are constants of integration. Following the procedure presented in appendix A, we can calculate the conductivity as

$$
\sigma=\frac{(3 z-2) r_{+}^{6 z-4} A^{1}}{4 \pi i \omega b^{2 z-2} l^{z+1} A^{0}}
$$

\subsubsection{Nonlinear electrodynamics}

Here we follow the above procedure to calculate the holographic conductivity in the case of nonlinear electrodynamics. We consider the four-dimensional planar metric

$$
d s^{2}=-\frac{\mathcal{F}(u) r_{+}^{4 z}}{l^{2 z} u^{2 z}} d t^{2}+\frac{l^{2} d u^{2}}{\mathcal{F}(u) u^{2}}+\frac{r_{+}^{4}}{u^{2}}\left(d x_{1}^{2}+d x_{2}^{2}\right),
$$

where

$$
\begin{aligned}
\mathcal{F}(u)= & 1-\frac{m u^{z+2}}{r_{+}^{2 z+4}}-\frac{2 \beta^{2} l^{2} b^{2 z-2} u^{2 z-2}}{(4-z) r_{+}^{4 z-4}}+\frac{2 \beta^{2} l^{2} b^{2 z-2} u^{z+2}}{r_{+}^{2 z+4}}\left(\frac{q^{2} l^{2 z-2}}{\beta^{2} L_{W}\left(\varrho_{u}\right)}\right)^{(4-z) / 4} \\
& \times\left\{\frac{L_{W}^{2}\left(\varrho_{u}\right)}{4+z} \mathbf{F}\left(\frac{4+z}{4}, \frac{8+z}{4}, \frac{z-2}{4} L_{W}\left(\varrho_{u}\right)\right)+\frac{1}{4-z} \mathbf{F}\left(\frac{z-4}{4}, \frac{z}{4}, \frac{z-2}{4} L_{W}\left(\varrho_{u}\right)\right)\right\},
\end{aligned}
$$

and $\varrho_{u} \equiv q^{2} l^{2 z-2} u^{4} / r_{+}^{8} \beta^{2}$. Turning on perturbations $g_{t x_{1}}(u) e^{-i \omega t}$ and $A_{x_{1}}(u) e^{-i \omega t}$, we find below equations of motion

$$
A_{x_{1}}^{\prime \prime}(u)+\left[\frac{\mathcal{F}^{\prime}(u)}{\mathcal{F}(u)}+\frac{3(1-z)}{u}+\frac{2 L_{W}\left(\varrho_{u}\right)}{u\left(1+L_{W}\left(\varrho_{u}\right)\right)}\right] A_{x_{1}}^{\prime}(u)
$$




$$
+\frac{l^{2 z+2} u^{2 z-2}}{r_{+}^{4 z} \mathcal{F}(u)^{2}}\left[\omega^{2} A_{x_{1}}(u)-\frac{\beta b^{2 z-2} u^{z-2} \mathcal{F}(u)}{l^{z+1} r_{+}^{2 z-4}} \sqrt{L_{W}\left(\varrho_{u}\right)}\left(2 g_{t x_{1}}(u)+u g_{t x_{1}}^{\prime}(u)\right)\right]=0,
$$

and

$$
2 g_{t x_{1}}(u)+u g_{t x_{1}}^{\prime}(u)=4 q r_{+}^{2 z-4} u^{2-z} A_{x_{1}}(u),
$$

The result of combining eqs. (3.17) and (3.18) is decoupled equation for $A_{x_{1}}$

$$
\begin{aligned}
& A_{x_{1}}^{\prime \prime}(u)+\left[\frac{\mathcal{F}^{\prime}(u)}{\mathcal{F}(u)}+\frac{3(1-z)}{u}+\frac{2 L_{W}\left(\varrho_{u}\right)}{u\left(1+L_{W}\left(\varrho_{u}\right)\right)}\right] A_{x_{1}}^{\prime}(u)+ \\
& \quad+\frac{l^{2 z+2} u^{2 z-2}}{r_{+}^{4 z} \mathcal{F}(u)^{2}}\left(\omega^{2}-\frac{4 q \beta b^{2 z-2} \mathcal{F}(u)}{l^{z+1}} \sqrt{L_{W}\left(\varrho_{u}\right)}\right) A_{x_{1}}(u)=0 .
\end{aligned}
$$

Near the $u=0$ boundary, eq. (3.19) reduces approximately to eq. (3.13) with the solution (3.14). Performing the procedure of appendix A, the holographic conductivity can be obtained as

$$
\sigma=\left.\frac{(3 z-2) r_{+}^{6 z-4} A^{1}}{4 \pi i \omega b^{2 z-2} l^{z+1} A^{0}} \exp \left[-\frac{1}{2}\left(\frac{l^{z} \omega A^{0} r_{+}^{2 z-6} u^{3-z}}{\beta b^{2 z-2}}\right)^{2}\right]\right|_{u=0}
$$

As one can see from (3.20), the behaviour of conductivity is different for different ranges of $z$

$$
\sigma= \begin{cases}\frac{(3 z-2) r_{+}^{6 z-4} A^{1}}{4 \pi i \omega b^{2 z-2} l^{z+1} A^{0}}, & \text { for } z<3 \\ \frac{(3 z-2) r_{+}^{6 z-4} A^{1}}{4 \pi i \omega b^{2 z-2} l^{z+1} A^{0}} \exp \left[-\frac{1}{2}\left(\frac{l^{z} \omega A^{0} r_{+}^{2 z-6}}{\beta b^{2 z-2}}\right)^{2}\right], & \text { for } z=3 \\ 0, & \text { for } z>3\end{cases}
$$

\subsubsection{Behavior of the conductivity and experimental results}

In order to illustrate the behavior of the conductivity, we should solve the decoupled differential equation for $A_{x_{1}}$. By defining

$$
A_{x_{1}}(u)=\mathcal{F}(u)^{-i 4 \pi \omega / T} S(u),
$$

near the horizon, where $S(u)=1+a\left(u-r_{+}\right)+b\left(u-r_{+}\right)^{2}+\cdots$, we remove the oscillations for numerical stability. It is remarkable to note that at the horizon where $S(u)=1$, $A_{x_{1}}(u) \propto \mathcal{F}(u)^{ \pm i 4 \pi \omega / T}$. However, we choose $\mathcal{F}(u)^{-i 4 \pi \omega / T}$ in (3.22) in order to perform ingoing boundary condition. The coefficients $a, b, \cdots$ can easily be found by looking for Taylor series expansion of differential equation (3.12) at the horizon. These coefficients are initial values necessary for solving eq. (3.12) numerically to obtain the conductivity.

Figures 13 and 14 depict the real and imaginary parts of the conductivity for $z=1$ in terms of $\omega / T$. It is notable to mention that the real part of the conductivity is the dissipative part while the imaginary part is the reactive one. Figure 13 shows that $\sigma_{D C} \equiv$ $\operatorname{Re}[\sigma(\omega=0)]$ is greater for smaller values of $q$. The same behavior can be seen for the nonlinear parameter $\beta$ i.e. $\sigma_{D C}$ increases when $\beta$ decreases. The behaviors of the real and imaginary parts of the conductivity are in excellent agreement with the experimental 


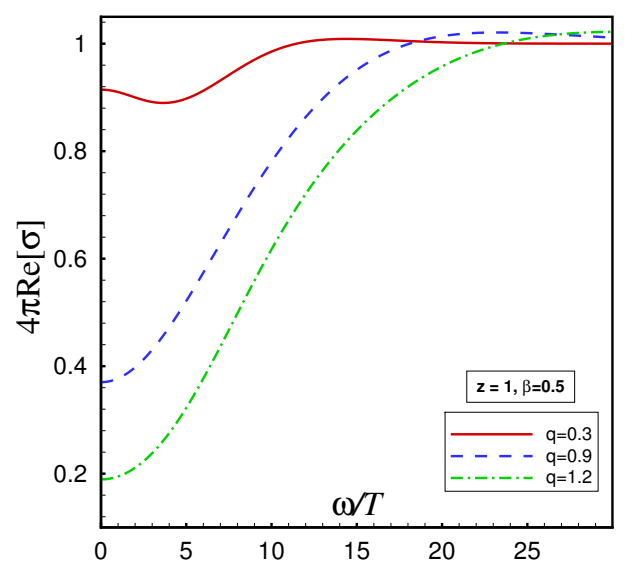

(a)

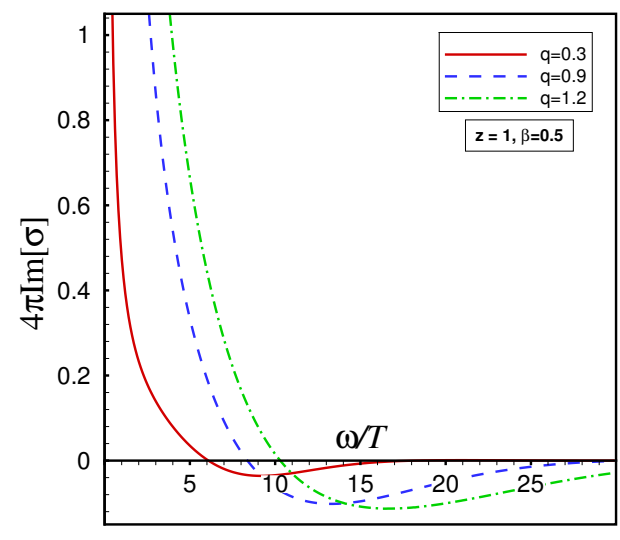

(b)

Figure 13. The behaviors of real and imaginary parts of conductivity $\sigma$ versus $\omega / T$ for $z=1$ and different values of $q$ with $l=b=r_{+}=1$.

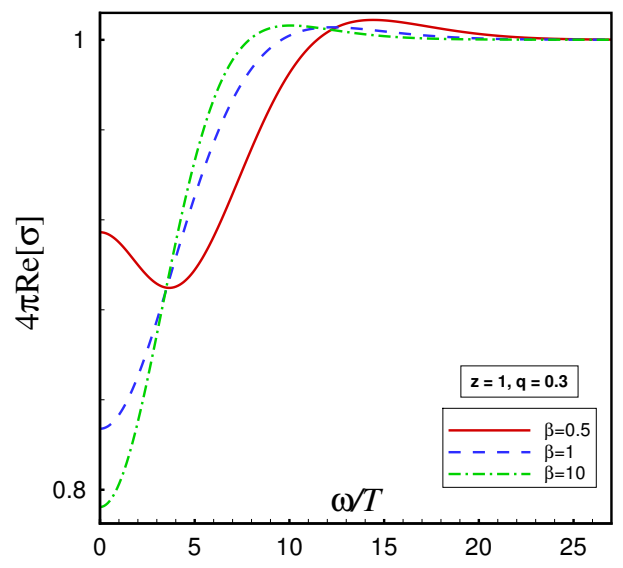

(a)

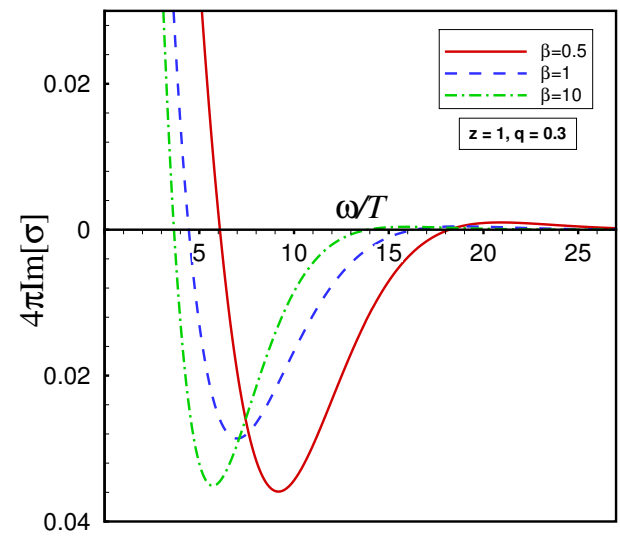

(b)

Figure 14. The behaviors of real and imaginary parts of conductivity $\sigma$ versus $\omega / T$ for $z=1$ and different values of $\beta$ with $l=b=r_{+}=1$.

results reported in [68]. These experimental results show the increase in $\operatorname{Re}[\sigma]$ near the zero frequency due to impurities and ionic lattice. The holographic conductivity computed by employing a gravity dual model with linear Maxwell electrodynamics cannot produce this behavior [69]. However, as one can see in figures 13 and 14, for suitable choices of parameters the nonlinearity of electrodynamics model can result this behavior for real part of conductivity.

The behaviors of the real and imaginary parts of the conductivity in terms of $\omega / T$ for $z=1.1$ are illustrated in figures 15 and 16. In this case, $\sigma_{D C}$ changes with respect 


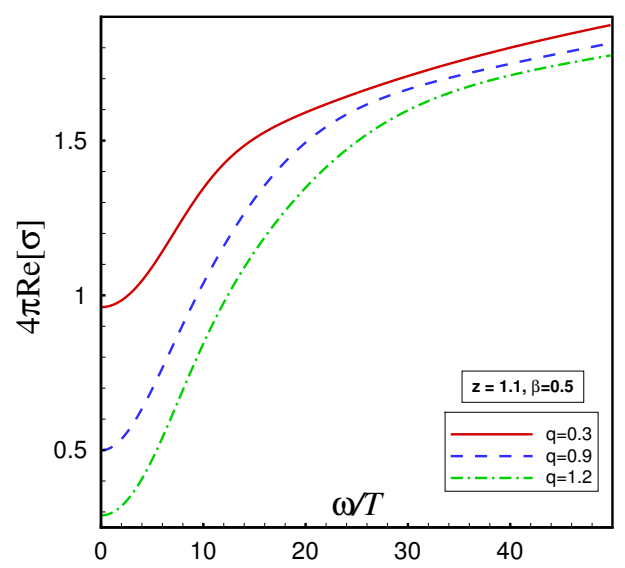

(a)

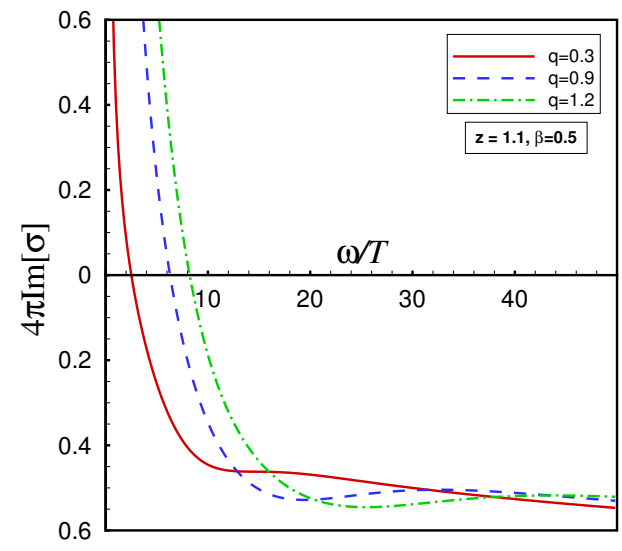

(b)

Figure 15. The behaviors of real and imaginary parts of conductivity $\sigma$ versus $\omega / T$ for $z=1.1$ and different values of $q$ with $l=b=r_{+}=1$.

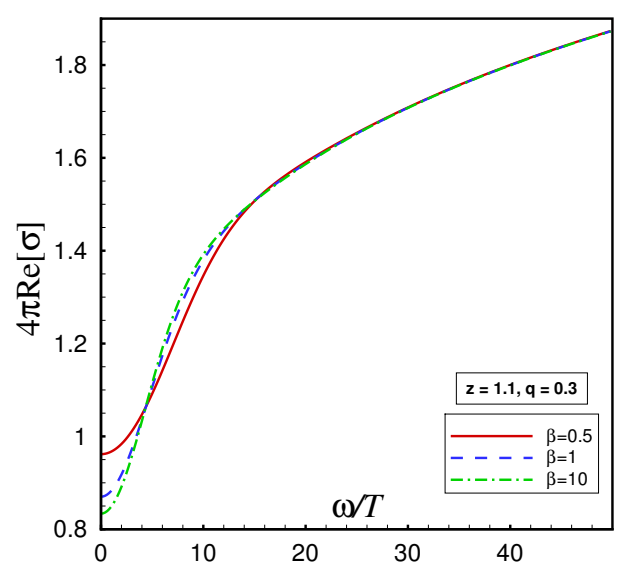

(a)

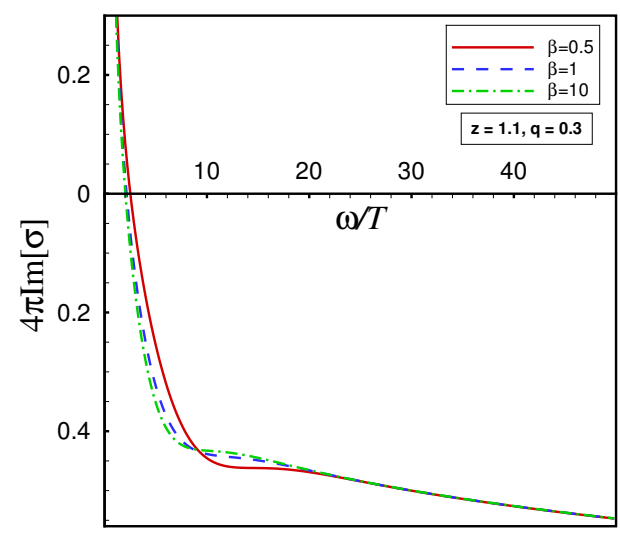

(b)

Figure 16. The behaviors of real and imaginary parts of conductivity $\sigma$ versus $\omega / T$ for $z=1.1$ and different values of $\beta$ with $l=b=r_{+}=1$.

to $q$ and $\beta$ in the similar manner that it does for $z=1$. However, in contrast to the asymptotic AdS spaces in which the real part of the conductivity tends to a constant in large frequencies, in this case $(z=1.1) \operatorname{Re}[\sigma]$ grows. Similar behavior for $\operatorname{Re}[\sigma]$ has been reported recently for the optical conductivity of single-layer graphene induced by mild oxygen plasma exposure [70]. It is remarkable to note that our numerical calculations show that there is no significant difference between the behaviour of conductivity for $\beta=10$ and larger. 


\section{Summary and concluding remarks}

In this paper, we constructed a new class of Einstein-dilaton-Lifshitz black solutions in the presence of exponential nonlinear electrodynamics. Our solutions respect the Lifshitz symmetry $t \rightarrow \lambda^{z} t$ and $\overrightarrow{\mathbf{x}} \rightarrow \lambda \overrightarrow{\mathbf{x}}$ at $r$-infinity boundary where $z(\geq 1)$ is the dynamical critical exponent. The exponential electrodynamics behaves as Born-Infeld electrodynamics for large values of the nonlinear parameter $\beta$ where they reduce to linear Maxwell regime, as expected. It is worth mentioning that, while the Born-Infeld nonlinear electrodynamics removes divergences in the electric field and has finite value near the origin where $r \rightarrow 0$, the exponential form of the nonlinear electromagnetics does not cancel the divergency of the electric field exactly at $r=0$, however, its singularity is much weaker than Maxwell theory. This is more reasonable compared to the Born-Infeld case, since near the origin where $r \rightarrow$ 0 , the electric field of a point-like charged particle should be an increasing function. The behavior of the electric field of the exponential nonlinear electrodynamics near the origin in the absence and in the presence of the dilaton field was explicitly shown in table A of refs. [46] and [51], respectively. Besides, it was argued that in applications of the AdS/CFT correspondence to superconductivity, exponential nonlinear electrodynamics, makes crucial effects on the condensation as well as the critical temperature of the superconductor [48]. It was also recently observed that, in the holographic superconductor, the exponential nonlinear electrodynamics can increase the critical values of the external magnetic field as the temperature goes to zero [49].

We considered topological black holes with zero $(k=0)$, positive $(k=1)$ and negative $(k=-1)$ horizon curvatures, but the reality of charge of asymptotic Lifshitz supporting Maxwell matter field imposes that there is no allowed solution with negative horizon curvature except for asymptotic $\operatorname{AdS}$ case $(z=1)$. Next, we calculated the conserved and thermodynamical quantities namely mass, charge, electric potential, entropy and temperature. We obtained the mass in terms of the extensive quantities entropy $s$ and charge $Q$ i.e. $M(s, Q)$ and checked that the intensive quantities temperature $T$ and electric potential $U$ calculated from it match with those obtained from the geometry of the black hole. Thus, the first law of thermodynamics is satisfied.

Subsequently, we turned out to study thermal stability of the obtained solutions. We showed that black brane solutions $(k=0)$ are always thermally stable in canonical ensemble. In grand-canonical ensemble black branes with horizon radius $r_{+}$larger than $r_{+\min }$ are stable while there is a maximum $z(\beta)$ that black branes are unstable for values greater than it. We observed that black holes $(k=1)$ encounter the Hawking-Page phase transition between small and large black holes in both canonical and grand-canonical ensembles. For $z \geq 2$, our black holes are always stable in canonical ensemble. For other values of $z$ in canonical ensemble as well as grand-canonical ensemble, there is a minimum for dynamical critical exponent that black holes are stable for values greater than it. Moreover, black holes are stable for nonlinear parameters greater than $\beta_{\min }$ in canonical ensemble while they are unstable for values greater than $\beta_{\max }$ in grand-canonical ensemble.

Afterward, we performed the gauge/gravity duality to compute the ratio of shear viscosity to entropy for a three-dimensional hydrodynamic system by using the pole method. 
We obtained the well-known $1 / 4 \pi$ result which shows that even the non-AdS symmetry of hydrodynamic system cannot affect the value of viscosity. Finally, we turned to study the behavior of the holographic conductivity for two-dimensional systems. We examined the issue for both linear Maxwell and nonlinear exponential electrodynamics. Our investigations revealed that the effect of nonlinearity is vanishing the conductivity for $z>3$. We depicted the behaviors of real and imaginary parts of conductivity for asymptotic AdS $(z=1)$ and Lifshitz cases and pointed out some two-dimensional graphene systems taken under consideration experimentally which have behaviors resemble our numerical results.

\section{Acknowledgments}

We thank from the Research Council of Shiraz University. This work has been supported financially by Research Institute for Astronomy \& Astrophysics of Maragha (RIAAM), Iran.

\section{A A brief review on gauge-gravity duality basics}

Here we are going to present a brief review on the idea of gauge-gravity duality (reader can see for instance [69] and [71] for more details). Let us start with QFT. The generating function in $\mathrm{QFT} Z_{\mathrm{QFT}}$ has a central role. For instance the expectation value of an operator $\mathcal{O}$ sourced by $\phi_{0}$ is given by

$$
\langle\mathcal{O}\rangle=\frac{1}{Z_{\mathrm{QFT}}\left[\phi_{0}\right]} \frac{\partial Z_{\mathrm{QFT}}\left[\phi_{0}\right]}{i \partial \phi_{0}}=\frac{\partial \ln \left(Z_{\mathrm{QFT}}\left[\phi_{0}\right]\right)}{i \partial \phi_{0}} .
$$

For a strongly interacting field theory, it is hard to compute the generating function $Z_{\mathrm{QFT}}$ and here is the position where gauge-gravity duality helps us. Consider a bulk that our field theory lives on its $u=0$ boundary. The function $\phi_{0}(x)$ on the boundary becomes a field $\phi(x, u)$ governed by an equation of motion in the bulk so that $\phi(x, u) \rightarrow \phi_{0}(x)$ as one approaches the boundary $u \rightarrow 0$. The fundamental formula of holography is GKPW formula (Gubser, Klebanov, Polyakov [72] and Witten [73]) that its classical gravity limit is

$$
Z_{\mathrm{QFT}}\left[\phi_{0}\right]=e^{i S_{\text {bulk }}}
$$

where $S_{\text {bulk }}$ is the action calculated by using solutions of bulk equations of motion obtained subject to the requirement that $\phi \rightarrow \phi_{0}$ on the boundary. Using (A.2), one can find that

$$
\langle\mathcal{O}\rangle=\frac{\partial S_{\text {bulk }}}{\partial \phi_{0}}
$$

In order to find a more convenient alternative for (A.3), we pause here to remind the Hamilton-Jacobi theory. Varying the action of a point particle with position $x$, one receives

$$
\delta S_{\text {particle }}=\int_{t_{i}}^{t_{f}} d t\left[\frac{\partial L}{\partial x}-\frac{d}{d t}\left(\frac{\partial L}{\partial \dot{x}}\right)\right] \delta x+\left[\frac{\partial L}{\partial \dot{x}} \delta x\right]_{t_{i}}^{t_{f}} .
$$

Considering the on-shell action and supposing that initial position of the particle is fixed $\left(\delta x\left(t_{i}\right)=0\right)$ while the final position is varying $\left(\delta x\left(t_{f}\right) \neq 0\right)$, one obtains

$$
\frac{\partial S}{\partial x_{f}}=\left.\frac{\partial L}{\partial \dot{x}}\right|_{t_{f}}=p_{f}
$$


We can generalize the above result to our case and rewrite (A.3) as

$$
\langle\mathcal{O}\rangle=\left.\frac{\partial \mathcal{L}}{\partial\left(\partial_{u} \phi\right)}\right|_{u=0}
$$

Therefore, we could obtain the expectation value of the operator $\mathcal{O}$ without calculating $Z_{\mathrm{QFT}}$. Note that the expectation value was a simple example to present the basic ideas of gauge-gravity duality. Another remark to note is that the operator $\mathcal{O}$ and its source $\phi_{0}$ which are scalars can be extended to vectors or tensors. For instance the source for $J^{i}$ is $A_{0}^{i}$ and the source for $T^{i j}$ is $g_{0}^{i j}$. As an example the expectation value of $J_{i}$ sourced by the perturbation $\delta A_{0 i}=A_{0 i} e^{-i \omega t}$ is

$$
\left\langle J_{i}\right\rangle=\left.\frac{\partial \mathcal{L}}{\partial\left(\partial_{u} \delta A_{i}\right)}\right|_{u=0}
$$

Then, with (A.7) in hand we can obtain the conductivity via the formula $\sigma=\left\langle J_{i}\right\rangle / E_{i}$.

Open Access. This article is distributed under the terms of the Creative Commons Attribution License (CC-BY 4.0), which permits any use, distribution and reproduction in any medium, provided the original author(s) and source are credited.

\section{References}

[1] R.P. Feynman, QED: the strange theory of light and matter, Princeton University Press, Princeton NJ U.S.A. (1988).

[2] J.M. Maldacena, The large- $N$ limit of superconformal field theories and supergravity, Int. J. Theor. Phys. 38 (1999) 1113 [hep-th/9711200] [INSPIRE].

[3] S.A. Hartnoll and P. Kovtun, Hall conductivity from dyonic black holes, Phys. Rev. D 76 (2007) 066001 [arXiv:0704.1160] [INSPIRE].

[4] M. Fujita, W. Li, S. Ryu and T. Takayanagi, Fractional quantum Hall effect via holography: Chern-Simons, edge states and hierarchy, JHEP 06 (2009) 066 [arXiv:0901.0924] [INSPIRE].

[5] S.A. Hartnoll, P.K. Kovtun, M. Muller and S. Sachdev, Theory of the Nernst effect near quantum phase transitions in condensed matter and in dyonic black holes, Phys. Rev. B 76 (2007) 144502 [arXiv:0706.3215] [INSPIRE].

[6] S.A. Hartnoll and C.P. Herzog, Ohm's law at strong coupling: $S$ duality and the cyclotron resonance, Phys. Rev. D 76 (2007) 106012 [arXiv:0706.3228] [InSPIRE].

[7] S.A. Hartnoll and C.P. Herzog, Impure AdS/CFT correspondence, Phys. Rev. D 77 (2008) 106009 [arXiv: 0801.1693] [INSPIRE].

[8] S.A. Hartnoll, C.P. Herzog and G.T. Horowitz, Building a holographic superconductor, Phys. Rev. Lett. 101 (2008) 031601 [arXiv: 0803.3295] [INSPIRE].

[9] S.A. Hartnoll, C.P. Herzog and G.T. Horowitz, Holographic superconductors, JHEP 12 (2008) 015 [arXiv:0810.1563] [INSPIRE].

[10] M. Ammon, J. Erdmenger, M. Kaminski and P. Kerner, Flavor superconductivity from gauge/gravity duality, JHEP 10 (2009) 067 [arXiv:0903.1864] [INSPIRE]. 
[11] G.T. Horowitz and M.M. Roberts, Holographic superconductors with various condensates, Phys. Rev. D 78 (2008) 126008 [arXiv:0810.1077] [InSPIRE].

[12] S.-J. Sin, S.-S. Xu and Y. Zhou, Holographic superconductor for a Lifshitz fixed point, Int. J. Mod. Phys. A 26 (2011) 4617 [arXiv:0909.4857] [INSPIRE].

[13] D.T. Son, Toward an AdS/cold atoms correspondence: a geometric realization of the Schrödinger symmetry, Phys. Rev. D 78 (2008) 046003 [arXiv: 0804.3972] [InSPIRE].

[14] A. Adams, K. Balasubramanian and J. McGreevy, Hot spacetimes for cold atoms, JHEP 11 (2008) 059 [arXiv: 0807.1111] [INSPIRE].

[15] C.P. Herzog, M. Rangamani and S.F. Ross, Heating up Galilean holography, JHEP 11 (2008) 080 [arXiv: 0807.1099] [INSPIRE].

[16] S. Kachru, X. Liu and M. Mulligan, Gravity duals of Lifshitz-like fixed points, Phys. Rev. D 78 (2008) 106005 [arXiv:0808.1725] [INSPIRE].

[17] G. Bertoldi, B.A. Burrington and A. Peet, Black holes in asymptotically Lifshitz spacetimes with arbitrary critical exponent, Phys. Rev. D 80 (2009) 126003 [arXiv:0905.3183] [INSPIRE].

[18] M.H. Dehghani and R.B. Mann, Lovelock-Lifshitz black holes, JHEP 07 (2010) 019 [arXiv: 1004.4397] [INSPIRE].

[19] M.H. Dehghani and R.B. Mann, Thermodynamics of Lovelock-Lifshitz black branes, Phys. Rev. D 82 (2010) 064019 [arXiv:1006.3510] [INSPIRE].

[20] M.H. Dehghani and S. Asnafi, Thermodynamics of rotating Lovelock-Lifshitz black branes, Phys. Rev. D 84 (2011) 064038 [arXiv:1107.3354] [INSPIRE].

[21] M.H. Dehghani, C. Shakuri and M.H. Vahidinia, Lifshitz black brane thermodynamics in the presence of a nonlinear electromagnetic field, Phys. Rev. D 87 (2013) 084013 [arXiv: 1306.4501] [INSPIRE].

[22] M. Bravo-Gaete and M. Hassaine, Thermodynamics of charged Lifshitz black holes with quadratic corrections, Phys. Rev. D 91 (2015) 064038 [arXiv:1501.03348] [INSPIRE].

[23] R.B. Mann, Lifshitz topological black holes, JHEP 06 (2009) 075 [arXiv:0905.1136] [INSPIRE].

[24] G. Bertoldi, B.A. Burrington and A.W. Peet, Thermal behavior of charged dilatonic black branes in AdS and UV completions of Lifshitz-like geometries, Phys. Rev. D 82 (2010) 106013 [arXiv: 1007.1464] [INSPIRE].

[25] J. Tarrio and S. Vandoren, Black holes and black branes in Lifshitz spacetimes, JHEP 09 (2011) 017 [arXiv: 1105.6335] [INSPIRE].

[26] M.K. Zangeneh, A. Sheykhi and M.H. Dehghani, Thermodynamics of topological nonlinear charged Lifshitz black holes, Phys. Rev. D 92 (2015) 024050 [arXiv:1506. 01784] [InSPIRE].

[27] M.K. Zangeneh, M.H. Dehghani and A. Sheykhi, Thermodynamics of Gauss-Bonnet-dilaton Lifshitz black branes, Phys. Rev. D 92 (2015) 064023 [arXiv: 1506.07068] [INSPIRE].

[28] M. Taylor, Non-relativistic holography, arXiv:0812.0530 [INSPIRE].

[29] D. Momeni, R. Myrzakulov, L. Sebastiani and M.R. Setare, Analytical holographic superconductors in AdS $S_{N}$-Lifshitz topological black holes, Int. J. Geom. Meth. Mod. Phys. 12 (2015) 1550015 [arXiv: 1210.7965] [INSPIRE]. 
[30] D. Roychowdhury, Lifshitz holography and the phases of the anisotropic plasma, arXiv: 1509.05229 [INSPIRE].

[31] D.-W. Pang, On charged Lifshitz black holes, JHEP 01 (2010) 116 [arXiv:0911.2777] [INSPIRE].

[32] A. Bhattacharyya and D. Roychowdhury, Lifshitz hydrodynamics and new massive gravity, arXiv: 1503.03254 [INSPIRE].

[33] S.A. Hartnoll, D.M. Hofman and D. Vegh, Stellar spectroscopy: Fermions and holographic Lifshitz criticality, JHEP 08 (2011) 096 [arXiv:1105.3197] [INSPIRE].

[34] J.P.S. Lemos and D.-W. Pang, Holographic charge transport in Lifshitz black hole backgrounds, JHEP 06 (2011) 122 [arXiv:1106.2291] [INSPIRE].

[35] Y. Bu, Holographic superconductors with $z=2$ Lifshitz scaling, Phys. Rev. D 86 (2012) 046007 [arXiv: 1211.0037] [INSPIRE].

[36] J.-W. Lu, Y.-B. Wu, P. Qian, Y.-Y. Zhao and X. Zhang, Lifshitz scaling effects on holographic superconductors, Nucl. Phys. B 887 (2014) 112 [arXiv:1311.2699] [INSPIRE].

[37] G. Tallarita, Holographic Lifshitz superconductors with an axion field, Phys. Rev. D 89 (2014) 106005 [arXiv:1402 .4691] [INSPIRE].

[38] D. Roychowdhury, Magnetoconductivity in chiral Lifshitz hydrodynamics, JHEP 09 (2015) 145 [arXiv: 1508. 02002] [INSPIRE].

[39] M. Born and L. Infeld, Foundations of the new field theory, Proc. Roy. Soc. Lond. A 144 (1934) 425 [INSPIRE].

[40] E.S. Fradkin and A.A. Tseytlin, Nonlinear electrodynamics from quantized strings, Phys. Lett. B 163 (1985) 123 [inSPIRE].

[41] E. Bergshoeff, E. Sezgin, C.N. Pope and P.K. Townsend, The Born-Infeld action from conformal invariance of the open superstring, Phys. Lett. B 188 (1987) 70 [InSPIRE].

[42] C.G. Callan Jr., C. Lovelace, C.R. Nappi and S.A. Yost, Loop corrections to superstring equations of motion, Nucl. Phys. B 308 (1988) 221 [INSPIRE].

[43] O.D. Andreev and A.A. Tseytlin, Partition function representation for the open superstring effective action: cancellation of Möbius infinities and derivative corrections to Born-Infeld Lagrangian, Nucl. Phys. B 311 (1988) 205 [INSPIRE].

[44] R.G. Leigh, Dirac-Born-Infeld action from Dirichlet $\sigma$-model, Mod. Phys. Lett. A 4 (1989) 2767 [INSPIRE].

[45] S.H. Hendi, Asymptotic charged BTZ black hole solutions, JHEP 03 (2012) 065 [arXiv: 1405.4941] [INSPIRE].

[46] S.H. Hendi, Asymptotic Reissner-Nordström black holes, Annals Phys. 333 (2013) 282 [arXiv: 1405.5359] [INSPIRE].

[47] S.H. Hendi, Thermodynamic properties of asymptotically Reissner-Nordström black holes, Annals Phys. 346 (2014) 42 [arXiv:1405.6996] [INSPIRE].

[48] Z. Zhao, Q. Pan, S. Chen and J. Jing, Notes on holographic superconductor models with the nonlinear electrodynamics, Nucl. Phys. B 871 (2013) 98 [arXiv:1212.6693] [INSPIRE].

[49] A. Sheykhi and Z. Abdollazadeh, External magnetic field in holographic superconductors with exponential nonlinear electrodynamics, in preparation. 
[50] A. Sheykhi and S. Hajkhalili, Dilaton black holes coupled to nonlinear electrodynamic field, Phys. Rev. D 89 (2014) 104019 [arXiv: 1504.04009] [InSPIRE].

[51] A. Sheykhi and A. Kazemi, Higher dimensional dilaton black holes in the presence of exponential nonlinear electrodynamics, Phys. Rev. D 90 (2014) 044028 [arXiv:1506.01786] [INSPIRE].

[52] M.K. Zangeneh, A. Sheykhi and M.H. Dehghani, Thermodynamics of topological nonlinear charged Lifshitz black holes, Phys. Rev. D 92 (2015) 024050 [arXiv:1506.01784] [INSPIRE].

[53] M. Abramowitz and I.A. Stegun, Handbook of mathematical functions, Dover, New York U.S.A. (1972).

[54] R.M. Corless, G.H. Gonnet, D.E.G. Hare, D.J. Jeffrey and D.E. Knuth, On the Lambert W function, Adv. Comput. Math. 5 (1996) 329 [INSPIRE].

[55] S.H. Hendi, A. Sheykhi and M.H. Dehghani, Thermodynamics of higher dimensional topological charged AdS black branes in dilaton gravity, Eur. Phys. J. C 70 (2010) 703 [arXiv: 1002.0202] [INSPIRE].

[56] J.D. Bekenstein, Black holes and entropy, Phys. Rev. D 7 (1973) 2333 [InSPIRE].

[57] S.W. Hawking, Black hole explosions, Nature 248 (1974) 30 [INSPIRE].

[58] G.W. Gibbons and S.W. Hawking, Cosmological event horizons, thermodynamics and particle creation, Phys. Rev. D 15 (1977) 2738 [InSPIRE].

[59] M.K. Zangeneh, A. Sheykhi and M.H. Dehghani, Thermodynamics of topological nonlinear charged Lifshitz black holes, Phys. Rev. D 92 (2015) 024050 [arXiv:1506.01784] [INSPIRE].

[60] S.W. Hawking, C.J. Hunter and D.N. Page, Nut charge, anti-de Sitter space and entropy, Phys. Rev. D 59 (1999) 044033 [hep-th/9809035] [InSPIRE].

[61] R.B. Mann, Misner string entropy, Phys. Rev. D 60 (1999) 104047 [hep-th/9903229] [INSPIRE].

[62] R.B. Mann, Entropy of rotating Misner string space-times, Phys. Rev. D 61 (2000) 084013 [hep-th/9904148] [INSPIRE].

[63] M. Cvetič and S.S. Gubser, Phases of $R$ charged black holes, spinning branes and strongly coupled gauge theories, JHEP 04 (1999) 024 [hep-th/9902195] [INSPIRE].

[64] M.M. Caldarelli, G. Cognola and D. Klemm, Thermodynamics of Kerr-Newman-AdS black holes and conformal field theories, Class. Quant. Grav. 17 (2000) 399 [hep-th/9908022] [INSPIRE].

[65] S.S. Gubser and I. Mitra, The evolution of unstable black holes in anti-de Sitter space, JHEP 08 (2001) 018 [hep-th/0011127] [INSPIRE].

[66] M.F. Paulos, Transport coefficients, membrane couplings and universality at extremality, JHEP 02 (2010) 067 [arXiv:0910.4602] [INSPIRE].

[67] R.C. Myers, M.F. Paulos and A. Sinha, Holographic studies of quasi-topological gravity, JHEP 08 (2010) 035 [arXiv: 1004.2055] [INSPIRE].

[68] Z.Q. Li et al., Dirac charge dynamics in graphene by infrared spectroscopy, Nature Phys. 4 (2008) 532 [arXiv:0807.3780].

[69] S.A. Hartnoll, Lectures on holographic methods for condensed matter physics, Class. Quant. Grav. 26 (2009) 224002 [arXiv:0903.3246] [INSPIRE]. 
[70] I. Santoso et al., Tunable optical absorption and interactions in graphene via oxygen plasma, Phys. Rev. B 89 (2014) 075134 [arXiv:1307.1358].

[71] D. Tong, Lectures on holographic conductivity, talk presented at Cracow school of theoretical physics, http://www.damtp.cam.ac.uk/user/tong/talks/zakopane.pdf, Poland (2013).

[72] S.S. Gubser, I.R. Klebanov and A.M. Polyakov, Gauge theory correlators from noncritical string theory, Phys. Lett. B 428 (1998) 105 [hep-th/9802109] [INSPIRE].

[73] E. Witten, Anti-de Sitter space and holography, Adv. Theor. Math. Phys. 2 (1998) 253 [hep-th/9802150] [INSPIRE]. 